\title{
SUR LA TORSION DES STRUCTURES DE CONTACT TENDUES
}

\author{
PAR VINCENT COLIN
}

ABSTRACT. - Thanks to torsion, an invariant introduced by E. Giroux, we give a sufficient topological condition for a 3-manifold to carry infinitely many non-isomorphic tight contact structures.

It is for example the case of every orientable Seifert fibered space over a surface of genus $g \geqslant 1$.

We also prove a rigidity result for the existence of some contact submanifolds in a universally tight contact 3-manifold. (C) 2001 Éditions scientifiques et médicales Elsevier SAS

RÉSUMÉ. - Grâce à la torsion, un invariant introduit par E. Giroux, on donne une condition topologique suffisante pour qu'une variété de dimension 3 porte une infinité de structures de contact tendues non isomorphes.

C'est par exemple le cas de tout fibré de Seifert orientable au-dessus d'une surface de genre $g \geqslant 1$.

On prouve également un résultat de rigidité pour l'existence de certaines sous-variétés de contact dans une variété de contact universellement tendue de dimension 3. ㅇ 2001 Éditions scientifiques et médicales Elsevier SAS

\section{Introduction}

Une structure de contact sur une variété $V$ de dimension 3 est la donnée d'un champ de plans $\xi$ de classe $\mathrm{C}^{1}$ qui, défini localement comme le noyau d'une 1-forme non singulière $\alpha$, vérifie en tout point : $\alpha \wedge \mathrm{d} \alpha \neq 0$. Remplacer la forme $\alpha$ par toute autre forme non singulière de noyau $\xi$ ne modifie pas le signe de la forme volume $\alpha \wedge \mathrm{d} \alpha$, si bien que toute structure de contact induit une orientation de la variété qui la porte.

La question abordée dans cet article est celle de la classification à difféomorphisme (resp. à isotopie) près de ces structures : on dit que deux structures de contact $\xi_{0}$ et $\xi_{1}$ définies sur une variété $V$ sont difféomorphes (resp. isotopes) s'il existe un difféomorphisme $\phi_{1}$ de $V$ (resp. un difféomorphisme $\phi_{1}$ de $V$ isotope à l'identité) tel que $\phi_{1 *} \xi_{0}=\xi_{1}$.

La première propriété permettant de distinguer des classes de conjugaison de structures de contact a été découverte par D. Bennequin [1] avant d'être exploitée de manière systématique par Y. Eliashberg [6] : une variété de contact $(V, \xi)$ est dite tendue si aucun disque plongé dans $V$ n'est tangent à $\xi$ en tous les points de son bord. Les structures qui ne sont pas tendues sont dites vrillées. On dit de plus que $(V, \xi)$ est universellement tendue si le rappel de $\xi$ dans le revêtement universel de $V$ est une structure tendue.

Le résultat de la classification des structures vrillées à isotopie près se révèle remarquablement simple. En effet, Y. Eliashberg [6] a démontré que, sur une variété fermée de dimension 3,

1991 MSC: 53C15

Mots Clés: variété de contact; structure tendue; torsion; tore incompressible 
deux structures de contact vrillées de même signe et homotopes comme champs de plans sont isotopes. En revanche, le monde des structures de contact tendues est riche de propriétés de rigidité géométrique.

\subsection{Torsion des structures tendues}

Dans [13,14], E. Giroux montre comment un nouvel invariant, la torsion, différencie les structures de contact tendues sur le tore $\mathbb{T}^{3}$.

DÉfINITION 1.1 (E. Giroux). - Si $(V, \xi)$ est une variété de contact, on associe à chaque classe d'isotopie $C$ de plongements du produit $\mathbb{T}^{2} \times[0,2 \pi]$ dans $V$ un entier naturel (éventuellement $+\infty)$, noté $\operatorname{Tor}(V, \xi, C)$, appelé la torsion de $\xi$ dans la classe $C$ et défini de la manière suivante : pour $n \geqslant 1$, on note $\xi_{n}$ la structure de contact définie sur $\mathbb{T}^{2} \times I=\mathbb{T}^{2} \times[0,2 \pi]=\{(x, y, t)\}$ par l'équation $\cos n t \mathrm{~d} x+\sin n t \mathrm{~d} y=0$ et $\operatorname{Tor}(V, \xi, C)$ le supremum de l'ensemble des entiers positifs $n$ pour lesquels il existe un plongement de contact de $\left(\mathbb{T}^{2} \times I, \xi_{n}\right)$ dans la classe $C$ de $(V, \xi)$. S'il n'existe pas de tel plongement, on pose $\operatorname{Tor}(V, \xi, C)=0$.

En bien des points, la torsion se présente comme une version contact du «width » de Gromov et des capacités en géométrie symplectique.

Ici, on se propose de montrer que cet invariant est effectif dans de nombreuses situations.

DÉFINITION 1.2. - Si $\phi, \psi: \mathbb{T}^{2} \rightarrow V$ sont deux plongements incompressibles du tore (injectifs $\operatorname{sur} \pi_{1}\left(\mathbb{T}^{2}\right)$ ), on dit que $\phi$ a une intersection persistante avec $\psi$ si, pour tout plongement $\phi^{\prime}$ isotope à $\phi$, l'intersection $\phi^{\prime}\left(\mathbb{T}^{2}\right) \cap \psi\left(\mathbb{T}^{2}\right)$ est non vide.

DÉFINITION 1.3. - Un plongement du tore $\phi: \mathbb{T}^{2} \rightarrow V$ est dit normal s'il existe un plongement $\psi: \mathbb{T}^{2} \rightarrow V$ tel que $\phi$ ait une intersection persistante avec $\psi$.

On étend ces définitions de manière naturelle au cas des plongements du tore épais $\mathbb{T}^{2} \times[0,1]$.

THÉORÈME 1.4. - Si $(V, \xi)$ est une variété de contact universellement tendue irréductible et si $C$ est une classe d'isotopie normale, alors $\operatorname{Tor}(V, \xi, C)<\infty$.

On démontre également des résultats de rigidité pour certaines sous-variétés de contact d'une variété de contact universellement tendue $(V, \xi)$.

DÉFINITION 1.5. - Un tore $T$ plongé dans une variété de contact $(V, \xi)$ est dit pré-lagrangien s'il existe une 1-forme $\alpha$ de noyau $\xi$ telle que, pour tout $x \in T,\left.\mathrm{~d} \alpha\right|_{\mathrm{T}_{x} T}=0$.

Dans [5], on montre que pour toute famille non vide de tores incompressibles disjoints dans une variété irréductible $V$ de dimension 3 , il existe une structure de contact universellement tendue sur $V$ qui trace un feuilletage en cercles sur chaque tore - lequel est alors automatiquement pré-lagrangien. Cependant, le fait pour un tore d'être pré-lagrangien est non générique et rare. De même que E. Giroux [13] a démontré que les tores lagrangiens dans le fibré cotangent de $\mathbb{T}^{2}$ muni de sa forme symplectique naturelle (différentielle de la forme de Liouville) sont tous isotopes à la section nulle, on obtient ici de fortes restrictions quant à la présence de tores pré-lagrangiens dans une classe d'isotopie donnée.

THÉORÈME 1.6. - Soient $(V, \xi)$ une variété de contact irréductible et universellement tendue et $\phi:\left(\mathbb{T}^{2} \times I, \xi_{1}\right) \rightarrow(V, \xi)$ un plongement de contact. Si $\psi: \mathbb{T}^{2} \rightarrow V$ est un plongement incompressible qui possède une intersection persistante avec $\phi$, alors aucun tore de $V$ isotope à l'image de $\psi$ n'est pré-lagrangien.

Comme corollaire, on montre :

$4^{\mathrm{e}}$ SÉRIE - TOME $34-2001-\mathrm{N}^{\circ} 2$ 
THÉORÈme 1.7. - Si $(V, \xi)$ est une variété de contact fermée (compacte sans bord), irréductible et universellement tendue, seulement un nombre fini de classes d'isotopies de sousvariétés de $V$ difféomorphes à $\mathbb{T}^{2} \times I$ contiennent une sous-variété de contact conjuguée à $\left(\mathbb{T}^{2} \times I, \xi_{1}\right)$.

\subsection{Construction de structures tendues}

Les résultats contenus dans cette sous-section reposent sur le théorème d'existence suivant, démontré dans [5], mais non encore publié. Sa preuve est fondée sur une combinaison de résultats de Y. Eliashberg et W. Thurston [9] et du théorème de chirurgie 2.8.

THÉORÈme 1.8 ([5]). - Si V est une variété orientable, irréductible, fermée et $T_{1}, \ldots, T_{n}$ une collection non vide de tores incompressibles disjoints, $V$ porte une structure de contact universellement tendue qui trace sur $T_{i}$, pour $i=1, \ldots, n$, un feuilletage en cercles.

On conjecture qu'une variété irréductible porte un nombre infini de structures de contact tendues si et seulement si elle contient un tore incompressible. En combinant le théorème 3.1 avec le théorème 1.8 , on démontre ici le résultat suivant :

THÉORÈME 1.9. - Si V est une variété orientable, fermée et irréductible de dimension 3 qui contient un tore incompressible situé dans une classe d'isotopie normale, alors $V$ porte une infinité de structures de contact universellement tendues deux à deux homotopes comme champs de plans mais deux à deux non isomorphes.

En particulier, on obtient :

THÉORÈME 1.10. - Tout fibré de Seifert orientable au-dessus d'une surface de genre $g \geqslant 1$ porte une infinité de structures de contact universellement tendues, deux à deux homotopes comme champs de plans mais deux à deux non isomorphes.

\section{Outils}

\section{1. Éléments de géométrie de contact}

\subsubsection{Courbe dans une variété de contact}

Une courbe $\gamma$ plongée dans une variété de contact $(V, \xi)$ est dite legendrienne si elle est tangente à $\xi$ en tout point. Dans la suite, on supposera que $\xi$ est une structure de contact coorientable. Si $S$ est une surface compacte orientée, plongée dans $V$ et de bord legendrien $\gamma$, on note $\operatorname{tb}(\gamma)$, l'invariant de Thurston-Bennequin de $\gamma$, défini comme l'enlacement entre $\gamma$ et la courbe $\gamma_{\varepsilon}$ obtenue en poussant légèrement $\gamma$ par un champ de vecteurs transverse à $\xi$. Sous ces hypothèses, si on note $\chi(S)$ la caractéristique de $S$ et si la structure $\xi$ est tendue, l'invariant de Thurston-Bennequin de la courbe $\gamma$ vérifie l'inégalité de Bennequin ([1] et [8]) :

$$
\operatorname{tb}(\gamma) \leqslant-\chi(S)
$$

De même, si $\gamma$ est transverse à la structure $\xi$, on définit l'auto-enlacement $\ell(\gamma)$ de $\gamma$ comme l'enlacement entre $\gamma$ et une courbe obtenue en poussant légèrement $\gamma$ selon une section non singulière de $\left.\xi\right|_{S}$.

La définition de l'invariant de Thurston-Bennequin s'étend au cas où $\gamma$ est une courbe legendrienne contenue dans une surface fermée incompressible (dont le $\pi_{1}$ s'injecte) $S$ : on note $\operatorname{tb}(\gamma, S)$ le degré, dans le fibré normal à $\gamma$ coorienté par $\dot{\gamma}$ et trivialisé par TS, d'une section 
transverse à $\xi$ et définie le long de $\gamma$. Dans ce cas, la définition dépend de la classe de $S$ à isotopie près.

Muni de cette définition, il est possible d'obtenir dans certains cas particuliers des inégalités du type de celle de Bennequin. Pour $n \in \mathbb{N} \backslash\{0\}$, on note $\xi_{n}$ la structure de contact définie sur le tore $\mathbb{T}^{3}=\left\{(x, y, t) \in(\mathbb{R} / 2 \pi \mathbb{Z})^{3}\right\}$ par l'équation : $\cos n t \mathrm{~d} x+\sin n t \mathrm{~d} y=0$. Pour tout $n \in \mathbb{N} \backslash\{0\}$, la structure $\xi_{n}$ est universellement tendue, car revêtue par la structure standard $\zeta$ de $\mathbb{R}^{3}=\{(x, y, t)\}$ d'équation $\cos t \mathrm{~d} x+\sin t \mathrm{~d} y=0$ qui est tendue d'après un théorème de Bennequin [1]. Dans ce contexte, voici l'un des ingrédient clé, dû à Y. Kanda [17], dont on se servira ultérieurement :

LEMME 2.1 ([17]). - Si T est un tore plongé dans $\left(\mathbb{T}^{3}, \xi_{n}\right)$ ou dans son revêtement $\left(\mathbb{R} \times \mathbb{S}^{1} \times \mathbb{S}^{1}, \xi_{n}\right)$, isotope au tore vertical d'équation $\{x=0\}$, et $\gamma$ est une courbe legendrienne, plongée dans $T$ et isotope à la courbe d'équation $\{x=0, y=0\}$, alors $\operatorname{tb}(\gamma, T) \leqslant-n$.

Ce résultat est une amélioration, dans le cas particulier de $\mathbb{T}^{3}$, du lemme :

Lemme 2.2. - Si $(V, \xi)$ est une variété de contact universellement tendue et $\phi: \mathbb{T}^{2} \rightarrow V$ est un plongement incompressible du tore, dont l'image contient une courbe fermée simple, legendrienne et non contractile $\gamma$, alors $\operatorname{tb}\left(\gamma, \phi\left(\mathbb{T}^{2}\right)\right) \leqslant 0$.

Démonstration. - Soit $p: E \rightarrow V$ le revêtement de $V$ dont le groupe fondamental est $2 \mathbb{Z}^{2} \subset$ $\phi_{*}\left(\pi_{1}\left(\mathbb{T}^{2}\right)\right) \simeq \mathbb{Z}^{2}$. Tout relevé compact $T$ de $\phi\left(\mathbb{T}^{2}\right)$ dans $E$ contient deux relevés de $\gamma$ qui bordent dans $T$ un anneau $A$. L'application $\left.p\right|_{\partial A}: \partial A \rightarrow \gamma$ est un revêtement de degré 4 et on a $: \operatorname{tb}(\partial A, A)=4 \operatorname{tb}\left(\gamma, \phi\left(\mathbb{T}^{2}\right)\right)$. Comme la structure $p^{*} \xi$ est tendue, l'inégalité de Bennequin s'applique : $\operatorname{tb}(\partial A, A) \leqslant-\chi(A)=0$.

\subsubsection{Feuilletage caractéristique des surfaces}

Génériquement, toute surface $S$ compacte et plongée dans une variété de contact $(V, \xi)$ est transverse à $\xi$ en dehors d'un nombre fini de points $\left(x_{i}\right)_{1 \leqslant i \leqslant n}$. Le choix d'une coorientation de $\xi$ près de $S$ et d'une orientation de $S$ fournit, sur $S \backslash\left(\bigcup_{1 \leqslant i \leqslant n} x_{i}\right)$, une orientation du champ de droites $\xi \cap \mathrm{T} S$. Celui-ci s'intègre alors en un feuilletage orienté, appelé feuilletage caractéristique de $S$ et noté $\xi S$. Les singularités de ce feuilletage, qui sont les points $\left(x_{i}\right)_{1 \leqslant i \leqslant n}$ où $\xi=\mathrm{T} S$, sont génériquement de deux types : elliptiques d'indice 1 ou hyperbolique d'indice -1 . Elles sont positives si les coorientations de $\xi$ et de $S$ coïncident et négatives sinon.

LEMME 2.3. - Soient $T$ un tore incompressible contenu dans une variété de contact tendue $(V, \xi)$ et $\gamma$ une courbe legendrienne fermée simple, contenue dans $T$. On suppose de plus qu'il existe un plongement $\phi: \mathbb{D}^{2} \times \mathbb{S}^{1} \rightarrow V$ tel que $\phi\left(\{0\} \times \mathbb{S}^{1}\right)=\gamma$ et que $\xi \phi\left(\partial \mathbb{D}^{2} \times \mathbb{S}^{1}\right)$ contienne une orbite périodique $\delta$, isotope à $\gamma$ dans l'image de $\phi$ par la restriction d'une isotopie $\left(f_{t}\right)_{t \in[0,1]}$ de $V$. Alors on $a: \operatorname{tb}(\gamma, T) \leqslant \operatorname{tb}\left(\delta, f_{1}(T)\right)$.

Démonstration. - On commence par remarquer qu'il existe un anneau $A$ de bord $\gamma \cup \delta$ transverse à $\phi\left(\partial \mathbb{D}^{2} \times \mathbb{S}^{1}\right)$. D'après l'inégalité de Bennequin, $\operatorname{tb}(\gamma \cup \delta, A) \leqslant 0$. Comme $A$ est transverse à $\phi\left(\partial \mathbb{D}^{2} \times \mathbb{S}^{1}\right)$, il vient que $\operatorname{tb}(\delta, A)=0$ et par suite, comme $\operatorname{tb}(\gamma \cup \delta, A)=$ $\operatorname{tb}(\gamma, A)+\operatorname{tb}(\delta, A)$, on obtient que $\operatorname{tb}(\gamma, A) \leqslant 0$.

On note alors $s$ une section transverse à $\xi$ le long de $\gamma$. Si $s^{\prime}$ est une section transverse à $\xi$ le long de $\delta$, le degré de $f_{1 *} s$ dans le fibré normal à $\delta=f_{1}(\gamma)$ trivialisé par $s^{\prime}$ vaut $\operatorname{tb}(\gamma, A)$. Ainsi, $\operatorname{tb}\left(\delta, f_{1}(T)\right)=\operatorname{tb}(\gamma, T)-\operatorname{tb}(\gamma, A)$ et la conclusion s'ensuit.

Les feuilletages caractéristiques de surfaces ont été étudiés en détail par E. Giroux. Il ressort de son travail le résultat suivant :

Lemme 2.4 ([11]). - Soient $(V, \xi)$ une variété de contact tendue, $D_{1}$ et $D_{2}$ deux disques disjoints dans la sphère $\mathbb{S}^{2}$ et $f: \mathbb{S}^{2} \rightarrow V$ un plongement tel que le feuilletage $\xi f\left(D_{i}\right)$ soit 
transverse à $\partial f\left(D_{i}\right)$ et ne contienne qu'une singularité elliptique, positive pour $i=1$ et négative pour $i=2$. Alors, pour tout voisinage $U$ de $f\left(\mathbb{S}^{2} \backslash\left(D_{1} \cup D_{2}\right)\right)$ dans $V$, il existe un plongement $f^{\prime}$ isotope à $f$, égal à $f$ sur $D_{1} \cup D_{2}$, dont l'image est incluse dans $f\left(\mathbb{S}^{2}\right) \cup U$ et tel que le feuilletage $\xi f^{\prime}\left(\mathbb{S}^{2} \backslash\left(D_{1} \cup D_{2}\right)\right)$ soit non singulier, toute feuille joignant $f^{\prime}\left(\partial D_{1}\right) \grave{a} f^{\prime}\left(\partial D_{2}\right)$.

Le résultat suivant se déduit directement du fait - outre la non-intégrabilité d'une structure de contact - qu'un feuilletage non singulier sur le tore possède une orbite périodique ou une orbite dense.

LEMME 2.5. - Tout tore $T$ plongé dans une variété de contact $(V, \xi)$ et transverse à $\xi$ peut être isotopé de manière $\mathrm{C}^{\infty}$-petite en un tore dont le feuilletage caractéristique possède une orbite périodique. De plus, cette modification peut être choisie constante à l'extérieur de tout disque fixé au préalable dans $T$.

Un résultat important sur lequel on va s'appuyer concerne la structure tendue $\zeta$ définie sur $U_{a}=\mathbb{R}^{2} \times[-a, a]=\{(x, y, \theta)\}$ (où $a$ est un réel strictement positif) par l'équation $\cos \theta \mathrm{d} x+\sin \theta \mathrm{d} y=0$.

Lemme 2.6 (voir aussi [4]). - Pour tout compact $C \subset U_{a}$, il existe une isotopie de $U_{a}$, égale à l'identité en dehors d'un compact $K$ de $\operatorname{Int} U_{a}$, qui déforme le plan $P_{0}=\mathbb{R}^{2} \times\{0\}$ en un plan $P_{1}$, et un disque $D$ inclus dans $P_{1}$, qui contient $C \cap P_{1}$, dont le bord est transverse à $\zeta$ et dont le feuilletage caractéristique ne contient qu'une singularité elliptique radiale positive $e_{+}$. De plus, parmi les feuilles issues de $e_{+}$, une a pour ensemble limite une singularité hyperbolique positive et toutes les autres ont pour ensemble limite une même singularité elliptique négative.

Démonstration. - On note, pour tout réel $r$ positif, $D_{r}$ le disque plongé dans $P_{0}$ et défini par :

$$
D_{r}=\left\{x^{2}+y^{2} \leqslant r, \theta=0\right\} .
$$

Si $r$ est assez grand, $C \subset D_{r} \times[-a, a]$. Pour un tel réel $r$ fixé, on note $f$ la feuille de $\xi P_{0}$ qui passe par le point de coordonnées $(0,0)$. Cette feuille intersecte $D_{r}$ et on note $f^{\prime}$ et $f^{\prime \prime}$ des segments contenus dans $f \backslash D_{r}$, de part et d'autre de $D_{r}$ (en suivant $f$, on rencontre d'abord $f^{\prime}$, puis $D_{r}$ et enfin $\left.f^{\prime \prime}\right)$.

On modifie alors $P_{0}$ en un plan $P_{0}^{\prime}$ par une isotopie $\mathrm{C}^{0}$-petite fixant la courbe $f$, laissant invariant le complémentaire d'un voisinage de $f^{\prime \prime}$ et dont le support ne rencontre pas $D_{r} \times[-a, a]$, pour faire apparaître le long de $f^{\prime \prime}$ sur $\zeta P_{0}^{\prime}$ deux singularités de signe négatif en position d'élimination (voir [7]). Les signes sont choisis de telle sorte qu'en venant de $D_{r}$ en suivant $f$, on rencontre d'abord la singularité elliptique $e_{-}$. Par une isotopie $\mathrm{C}^{0}$-petite de $P_{0}^{\prime}$ fixant $f$, on fait de même apparaitre le long de $f^{\prime}$ sur le feuilletage du nouveau plan $P_{0}^{\prime \prime}$ deux singularités positives en position d'élimination. On choisit le support de cette deuxième isotopie assez petit pour que les orbites issues de la singularité elliptique positive $e_{+}$aient pour ensemble limite $e_{-}$, à l'exception de celle qui relie $e_{+}$et la singularité hyperbolique positive (cf. figure 1).

Si $\varepsilon \in \mathbb{R}^{+*}$ est choisi suffisamment petit, $D_{\varepsilon} \subset D_{r}$ et toute feuille passant par $D_{\varepsilon}$ contient $e_{+}$ dans son ensemble $\omega_{-}$-limite.

Sur $U_{a}$, la structure $\zeta$ est invariante par les difféomorphismes

$$
\phi_{\lambda}:(x, y, \theta) \longmapsto(\lambda x, \lambda y, \theta) .
$$

En prenant l'image de $P_{0}^{\prime \prime}$ par $\phi_{r / \varepsilon}$, on obtient un plan $P_{1}$ contenant $D_{r}=\phi_{r / \varepsilon}\left(D_{\varepsilon}\right)$, tel que toutes les courbes de $\zeta P_{1}$ passant par $D_{r}$ partent de la singularité elliptique $\phi_{r / \varepsilon}\left(e_{+}\right)$du feuilletage de $P_{1}$. De plus, $P_{1} \cap D_{r} \times[-a, a]=D_{r}$ et donc $P_{1} \cap C \subset D_{r}$. 


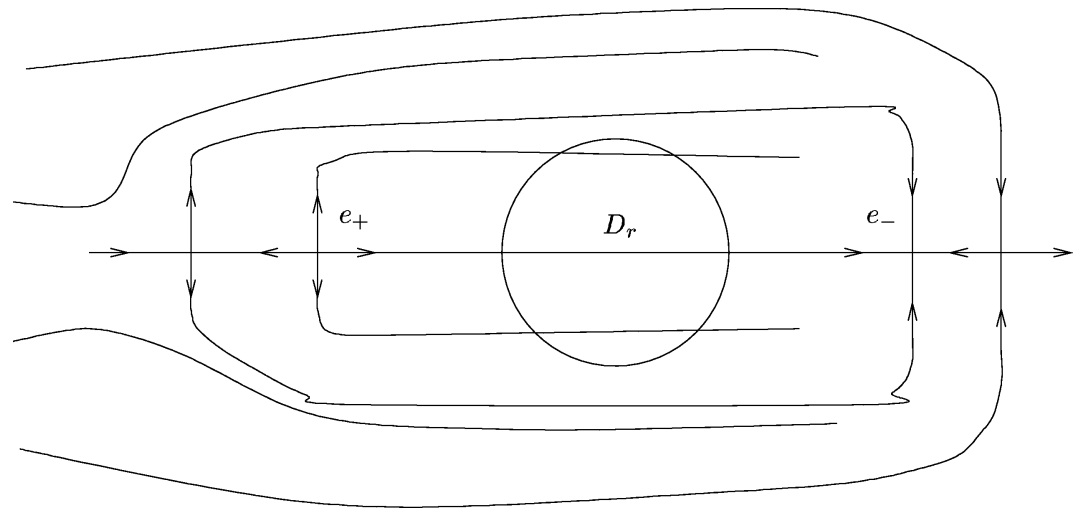

Fig. 1. Le feuilletage de $P_{1}$.

On peut alors trouver un disque $D$ plongé dans $P_{1}$, contenant $D_{r}$, de bord transverse au feuilletage caractéristique de $P$ et contenant la singularité elliptique $\phi_{r / \varepsilon}\left(e_{+}\right)$.

Lemme 2.7. - Soit $\xi$ une structure de contact sur $\mathbb{D}^{2} \times[-1,1]$ qui trace sur $\mathbb{D}^{2} \times\{0\}$ un feuilletage comprenant une singularité elliptique radiale $e_{+}$. On suppose de plus que, parmi les feuilles de $\xi \mathbb{D}^{2} \times\{0\}$ issues de $e_{+}$, une a pour ensemble limite une singularité hyperbolique positive et toutes les autres ont pour ensemble limite une même singularité elliptique radiale négative $e_{-}$. Si $\gamma$ est un segment legendrien dans $\left(\mathbb{D}^{2} \times[-1,1], \xi\right)$ qui va de $\mathbb{D}^{2} \times\{-1\}$ à $\mathbb{D}^{2} \times\{1\}$ et qui rencontre $\mathbb{D}^{2} \times\{0\}$ transversalement selon un point $z$ situé sur une feuille reliant $e_{+} \grave{a} e_{-}$, alors pour tout point $z^{\prime}$ situé sur une feuille reliant $e_{+} \grave{a} e_{-}$, il existe une isotopie legendrienne $\left(\gamma_{t}\right)_{t \in[0,1]}$ de $\gamma\left(\gamma_{0}=\gamma\right)$, fixe près de $\partial \gamma$ et telle que $\gamma_{1}$ intersecte transversalement $\mathbb{D}^{2} \times\{0\}$ en $z^{\prime}$.

Démonstration. - Il existe deux feuilles de $\xi \mathbb{D}^{2} \times\{0\}$ reliant $e_{+}$à $e_{-}$, dont la réunion borde dans $\mathbb{D}^{2} \times\{0\}$ un disque $D$ contenant $z$ et $z^{\prime}$ dans son intérieur. Ce disque (singulier) $D$ est feuilleté par des liaisons entre $e_{+}$et $e_{-}$. Comme le germe d'une structure de contact au voisinage d'une surface est entièrement déterminé par le feuilletage caractéristique qu'elle y induit ([11]), au voisinage de $D \backslash\left(e_{+} \cup e_{-}\right)$, la structure $\xi$ est conjugée à : $([-\varepsilon, \varepsilon] \times[0,1] \times]-\pi, \pi[=$ $\{(x, y, t)\}, \operatorname{ker}(\cos t \mathrm{~d} x+\sin t \mathrm{~d} y))$, avec $D \backslash\left(e_{+} \cup e_{-}\right)=\{x=0\}$.

Si $\varepsilon^{\prime}>0$ est choisi suffisamment petit (et $\varepsilon^{\prime}<\varepsilon$ ), pour tout $t \in\left[-\varepsilon^{\prime}, \varepsilon^{\prime}\right]$, le segment $\gamma$ rencontre transversalement le disque $\{x=t\}$ selon un point. On note alors

$$
\left.p:\left[-\varepsilon^{\prime}, \varepsilon^{\prime}\right] \times[0,1] \times\right]-\pi, \pi\left[\rightarrow\left[-\varepsilon^{\prime}, \varepsilon^{\prime}\right] \times[0,1]\right.
$$

la projection parallèle à $\partial / \partial t$. On trace une famille continue de segments $\left(\alpha_{s}\right)_{s \in[0,1]}$ dans $\left[-\varepsilon^{\prime}, \varepsilon^{\prime}\right] \times[0,1]$ avec les propriétés suivantes :

$-\alpha_{0}=p(\gamma)$,

- $\alpha_{s}$ coïncide avec $p(\gamma)$ au voisinage de $\left\{x= \pm \varepsilon^{\prime}\right\}$,

- pour tout $t \in\left[-\varepsilon^{\prime}, \varepsilon^{\prime}\right], \alpha_{s}$ est transverse à $\{x=t\}$,

- $\alpha_{1} \cap\{x=0\}=p\left(z^{\prime}\right)$ et $\mathrm{T}_{p\left(z^{\prime}\right)} \alpha_{1}=\operatorname{ker}\left(\cos t^{\prime} \mathrm{d} x+\sin t^{\prime} \mathrm{d} y\right.$ ) (où $z^{\prime}=\left(x^{\prime}, y^{\prime}, t^{\prime}\right)$ ).

En tout point $(x, y) \in\left[-\varepsilon^{\prime}, \varepsilon^{\prime}\right] \times[0,1]$, on a

$$
\mathrm{T}_{(x, y)} \alpha_{s}=\operatorname{ker}\left(\cos \left(f_{s}(x, y)\right) \mathrm{d} x+\sin \left(f_{s}(x, y)\right) \mathrm{d} y\right),
$$


et on note $\left.\gamma_{s} \subset[-\varepsilon, \varepsilon] \times[0,1] \times\right]-\pi, \pi\left[\right.$ le segment égal à $\gamma$ hors de $\left.\left[-\varepsilon^{\prime}, \varepsilon^{\prime}\right] \times[0,1] \times\right]-\pi, \pi[$ et qui est $\left\{\left(x, y, f_{s}(x, y)\right)\right\}$ à l'intérieur. Par construction, pour tout $s \in[0,1], \gamma_{s}$ est legendrien, $\gamma_{0}=\gamma$ et $\gamma_{1}$ rencontre $\{x=0\}$ transversalement selon $z^{\prime}$. C'est l'isotopie recherchée.

On rappelle enfin un résultat de chirurgie pour les variétés de contact tendues :

THÉORÈME 2.8 ([4]). - Soient $(V, \xi)$ une variété de contact et

$$
\phi:\left(U^{\prime}=\mathbb{T}^{2} \times[-\varepsilon, \varepsilon]=\{(x, y, t)\}, \operatorname{ker}(\cos t \mathrm{~d} x+\sin t \mathrm{~d} y)\right) \longrightarrow(V, \xi)
$$

un plongement de contact incompressible tels que $V \backslash \phi\left(U^{\prime}\right)$ soit universellement tendue. Alors, $(V, \xi)$ est universellement tendue. Cette conclusion vaut également pour tout plongement incompressible propre

$$
\phi:\left(U^{\prime}=\mathbb{S}^{1} \times \mathbb{R} \times[-\varepsilon, \varepsilon]=\{(x, y, t)\}, \operatorname{ker}(\cos t \mathrm{~d} x+\sin t \mathrm{~d} y)\right) \longrightarrow(V, \xi) .
$$

On fixe par ailleurs la notation suivante : si $S$ est une surface orientée, plongée dans $V$, on note

$$
V \backslash S
$$

la variété à bord obtenue en découpant $V$ le long de $S$. On a alors une application naturelle $\pi: V \imath S \rightarrow V$ qui est un difféomorphisme sur $V \backslash S$ et qui induit le revêtement d'orientation sur $S$.

Comme le germe d'une structure de contact $\xi$ au voisinage d'une surface $S$ est déterminé, à isotopie près, par le feuilletage caractéristique $\xi S$, toute structure de contact $\xi$ sur $V \imath S$ qui trace sur les deux composantes de $\partial(V \nmid S)$ deux feuilletages qui ont même image par $\pi$ est isotope, relativement à $\partial(V \nmid S)$, à une structure $\xi^{\prime}$ qui est la préimage par $\pi$ d'une structure de contact définie sur $V$.

\subsection{Outils topologiques}

\subsubsection{Isotopies de surfaces}

On rappelle une définition classique en topologie de dimension 3 :

DÉfinition 2.9. - Soient $F$ et $G$ deux surfaces dans $V$. On dit que $F$ est parallèle à $G$ s'il existe une surface $H$ et une application $f: H \times[0,1] \rightarrow V$, telles que :

- $\left.f\right|_{\operatorname{Int}(H \times[0,1])}$ soit un plongement,

- $\left.f\right|_{H \times\{0\}}$ soit un homéomorphisme sur $F$,

- $\left.f\right|_{\partial(H \times[0,1]) \backslash \operatorname{Int}(H \times\{0\})}$ soit un homéomorphisme sur $G$.

On extrait des travaux de F. Waldhausen (proposition 5.4 dans [18]) le résultat suivant :

THÉORÈME 2.10. - Soient $V$ une variété de dimension 3 irréductible et $F$ et $G$ des surfaces incompressibles et transverses dans $V$. On suppose qu'il existe une surface $H$ et une application continue $f: H \times[0,1] \rightarrow V$, telle que $\left.f\right|_{H \times\{0\}}$ soit un revêtement de $F$ et que $f(H \times\{1\}) \subset G$. Alors il existe une surface $\widetilde{F} \subset F$ et une surface $\widetilde{G} \subset G$ qui sont parallèles dans $V$ et, de plus, $\widetilde{F} \cap G=\partial \widetilde{F}$ et, ou bien $\widetilde{G} \cap F=\partial \widetilde{G}$, ou bien $\widetilde{G}$ et $\widetilde{F}$ sont des disques.

Voici des corollaires de ce résultat.

Lemme 2.11. - Soient $V$ une variété de dimension 3 irréductible et $F, G$ et $H_{1}, \ldots, H_{n}$ des tores incompressibles plongés dans $V$ tels qu'il existe dans $V$ une isotopie de disjonction de $F$ et $G$ et que, pour $i=1, \ldots, n, H_{i} \cap(F \cup G)=\emptyset$. Alors il existe une isotopie de disjonction de $F$ et $G$ dans $V \backslash\left(\bigcup_{i} H_{i}\right)$. 
Démonstration. - On commence par éliminer les composantes homotopiquement triviales de $F \cap G$ par une isotopie de $G$ dont l'image est contenue dans $V \backslash\left(\bigcup_{i} H_{i}\right)$.

Soit $D \subset F$ un disque intérieur à $F$ (i.e. tel que $D \cap G=\partial D$ ). Comme $G$ est incompressible, $\partial D$ borde un disque $D^{\prime}$ dans $G$ et comme $D$ est intérieur, la sphère $D \cup D^{\prime}$ est plongée. Or, $V$ est irréductible et donc $D \cup D^{\prime}$ borde une boule $B$ dans $V$, telle que $B \cap G=D^{\prime}$. Comme, pour $i=1, \ldots, n$, le tore $H_{i}$ ne rencontre ni $F$ ni $G$, il ne rencontre pas $B$. On pousse alors $D^{\prime}$ à travers $D$ dans $B$, ce qui permet d'éliminer les composantes de $F \cap G$ contenues dans $D^{\prime}$. On conclut cette première partie par récurrence.

On suppose à présent que toutes les composantes de $F \cap G$ sont non contractiles. Soit $F^{\prime}$ un tore isotope à $F$, transverse à $F$ et disjoint de $G$. D'après le théorème 2.10, il existe une surface $\widetilde{F} \subset F$ et une surface $\widetilde{F}^{\prime} \subset F^{\prime}$ qui sont parallèles dans $V$. On sait de plus que $\widetilde{F} \cap F^{\prime}=\partial \widetilde{F}$ et que, ou bien $\widetilde{F}^{\prime} \cap F=\partial \widetilde{F^{\prime}}$, ou bien $\widetilde{F}$ et $\widetilde{F^{\prime}}$ sont des disques. On note $P$ le produit plongé entre $\widetilde{F}$ et $\widetilde{F^{\prime}}$.

- Si $G$ rencontre $P$, comme $G$ ne rencontre pas $F^{\prime}$, l'intersection de $G$ et $\partial P$ est incluse dans $F$. Comme de plus les composantes de $F \cap G$ sont non contractiles, $\widetilde{F}$ n'est pas un disque et donc Int $P \cap\left(F \cup F^{\prime}\right)=\emptyset$. Le théorème des surfaces parallèles (proposition 3.1 dans [18]) affirme qu'il existe $\widehat{F} \subset \widetilde{F}$ et $\widehat{G} \subset G$ qui sont parallèles dans $P$. Comme, pour $i=1, \ldots, n$, le tore $H_{i}$ est incompressible et ne rencontre ni $F$ ni $G$, il ne rencontre pas non plus le produit contenu entre $\widehat{F}$ et $\widehat{G}$. Ainsi, l'isotopie de $G$ qui consiste à pousser $\widehat{G}$ au travers de $\hat{F}$ a une image qui ne rencontre pas $H_{i}$ et fait baisser le nombre de composantes de $F \cap G$.

- Si $G$ ne rencontre pas $P$, alors on pousse $\widetilde{F}^{\prime}$ au travers de $\widetilde{F}$ pour obtenir un tore $F^{\prime \prime}$ disjoint de $G$ dont le nombre de composantes d'intersection avec $F$ est moindre que celui de $F^{\prime}$.

On recommence alors le raisonnement précédent jusqu'à éliminer toutes les composantes de $F \cap G$ ou toutes les composantes de $F \cap F^{\prime}$. Dans ce dernier cas, $F$ et le nouveau tore $F^{\prime \prime}$ bordent un produit et une nouvelle application du théorème des surfaces parallèles permet d'éliminer les composantes de $F \cap G$ restantes.

LEMME 2.12. - Soient $\phi: \mathbb{T}^{2}=\mathbb{S}^{1} \times \mathbb{S}^{1} \rightarrow V$ un plongement incompressible dans une variété irréductible $V$ et $f: \mathbb{D}^{2} \times \mathbb{S}^{1} \rightarrow V$ un plongement dont l'image $T$ contient $\phi\left(\{0\} \times \mathbb{S}^{1}\right)$ dans son intérieur. On suppose de plus que la classe de $\phi\left(\{0\} \times \mathbb{S}^{1}\right)$ engendre $\pi_{1}(T)$. Alors il existe une isotopie $\left(\phi_{t}\right)_{t \in[0,1]}$ de $\phi$ telle que $\phi_{1}\left(\{0\} \times \mathbb{S}^{1}\right)=f\left(0 \times \mathbb{S}^{1}\right)$ et que pour tout $t \in[0,1]$, $\phi_{t}\left(\{0\} \times \mathbb{S}^{1}\right) \subset T$.

Démonstration. - On effectue une isotopie de $\phi$ qui rend l'image du nouveau plongement $\phi^{\prime}$ transverse au bord de $T$ et laisse $\phi^{\prime}\left(\{0\} \times \mathbb{S}^{1}\right)$ dans l'intérieur de $T$. On note alors $A$ l'adhérence de la composante de $\phi^{\prime}\left(\mathbb{T}^{2}\right) \backslash \partial T$ qui contient $\phi^{\prime}\left(\{0\} \times \mathbb{S}^{1}\right)$. Deux composantes de $\partial A$ sont non contractiles dans $V$ et les autres sont contractiles dans $\partial T$.

Comme de plus $V$ est irréductible, il existe une isotopie de $\phi^{\prime}$ en un plongement $\phi^{\prime \prime}$, fixe au voisinage de $\{0\} \times \mathbb{S}^{1}$ et qui élimine les composantes homotopiquement triviales de $\partial A$. Pour démontrer cette affirmation, on note $F$ l'image de $\phi^{\prime}$ et $D \subset \partial T$ un disque intérieur à $\partial T$ (i.e. tel que $D \cap F=\partial D$ ). Le cercle $\partial D$ borde dans $F$ un disque $D^{\prime}$. Comme $D$ est intérieur, la sphère $D \cup D^{\prime}$ est plongée dans $V$ qui est irréductible. Elle borde donc une boule $B$ dans $V$, telle que $B \cap F=D^{\prime}$. La boule $B$ ne rencontre pas la courbe $\phi^{\prime}\left(\{0\} \times \mathbb{S}^{1}\right)$. On pousse alors $D^{\prime}$ à travers $D$ dans $B$, ce qui permet d'éliminer les composantes de $\partial T \cap F$ contenues dans $D^{\prime}$. On conclut par récurrence.

La nouvelle surface $A^{\prime}$ est un anneau dont le bord est dans $\partial T$. Elle contient la courbe $\phi^{\prime \prime}\left(\{0\} \times \mathbb{S}^{1}\right)$ dont la classe engendre le groupe fondamental de $T$. En particulier, chaque composante de $\partial A^{\prime}$ a une intersection égale à \pm 1 avec un méridien de $\partial T$. D'après la proposition 3.1 de [18], l'anneau $A^{\prime}$ découpe $T$ en deux tores pleins et peut donc être poussé 
dans $\partial T$ par une isotopie qui fixe son bord. L'image de $\phi^{\prime \prime}\left(\{0\} \times \mathbb{S}^{1}\right)$ par cette isotopie est une courbe plongée dans $\partial T$ qui a une intersection égale à \pm 1 avec le méridien. Elle peut donc être isotopée à $f\left(0 \times \mathbb{S}^{1}\right)$.

LEMME 2.13. - Deux plans disjoints et proprement plongés dans $\mathbb{R}^{3}$ bordent dans $\mathbb{R}^{3}$ une sous-variété conjuguée à $\mathbb{R}^{2} \times[0,1]$.

Démonstration. - On donne la démarche à suivre.

On note $P$ et $Q$ deux plans disjoints et proprement plongés dans $\mathbb{R}^{3}$. On compactifie $\mathbb{R}^{3}$ en $\mathbb{S}^{3}$ par ajout d'un point $\infty$ de telle sorte que l'image $S_{P}$ de $P$ soit une sphère lisse passant par $\infty$. L'image $S_{Q}$ de $Q$ dans $\mathbb{S}^{3}$ est homéomorphe à une sphère et est lisse en dehors de $\infty$. On se donne alors une base de voisinages de $\infty$ formée d'une suite $\left(B_{n}\right)_{n \in \mathbb{N}}$ de boules emboîtées qui rencontre $S_{P}$ transversalement selon une famille $\left(D_{n}\right)_{n \in \mathbb{N}}$ de disques emboîtés. Si on fait l'hypothèse que, pour $0 \leqslant i \leqslant n$, la boule $B_{i}$ rencontre $S_{Q}$ transversalement selon un disque $D_{i}^{\prime}$, on applique le théorème 2.10 à $S_{Q}$ et $\partial B_{n+1}$ pour isotoper $S_{Q}$, par une isotopie fixe près de $\infty$ et de $S_{Q} \backslash D_{n}^{\prime}$ en une nouvelle sphère qui rencontre transversalement $B_{n+1}$ selon un disque $D_{n+1}^{\prime}$, et $S_{P}$ en $\infty$. On montre ainsi par récurrence que $S_{Q}$ peut être isotopée en une sphère $S_{Q}^{\prime}$ avec les propriétés suivantes :

- $S_{Q}^{\prime}$ est lisse en dehors de $\infty$,

$-S_{Q}^{\prime} \cap S_{P}=\infty$,

- pour tout $n \in \mathbb{N}, S_{Q}^{\prime}$ rencontre transversalement $B_{n}$ selon un disque $D_{n}^{\prime}$.

Les cercles $\partial D_{n}$ et $\partial D_{n}^{\prime}$ bordent un anneau $A_{n}$ dans la sphère $\partial B_{n}$. La sphère $D_{n} \cup A_{n} \cup D_{n}^{\prime}$ borde dans $\mathbb{S}^{3}$ une boule donnée par l'image d'un plongement $\phi_{n}: \mathbb{D}^{n} \times[0,1] \rightarrow \mathbb{S}^{3}$ tel que, si $\mathbb{D}^{n}$ désigne le disque de centre 0 et de rayon $n+1$ dans $\mathbb{R}^{2}$,

$\phi_{n}\left(\mathbb{D}^{n} \times\{0\}\right)=D_{n}, \quad \phi_{n}\left(\mathbb{D}^{n} \times\{1\}\right)=D_{n}^{\prime}, \quad \phi_{n}\left(\partial \mathbb{D}^{n} \times[0,1]\right)=A_{n}$ et $\left.\phi_{n+1}\right|_{\mathbb{D}^{n} \times[0,1]}=\phi_{n}$.

On obtient ainsi une structure produit entre $S_{P} \backslash \infty$ et $S_{Q}^{\prime} \backslash \infty$ et donc entre $S_{P} \backslash \infty$ et $S_{Q} \backslash \infty$. On en déduit que dans $\mathbb{R}^{3}$, les plans $P$ et $Q$ bordent un produit.

\subsubsection{Intersection persistante de tores incompressibles}

Voici à présent des résultats plus spécifiques.

Lemme 2.14. - Soient $V$ une variété de dimension 3 revêtue par $\mathbb{R}^{3}, \phi, \psi: \mathbb{T}^{2} \rightarrow V$ deux plongements incompressibles du tore, d'images respectives $F$ et $G$ et $x \in F \cap G$.

Si $\phi_{*}\left(\pi_{1}\left(\mathbb{T}^{2}, \phi^{-1}(x)\right)\right) \cap \psi_{*}\left(\pi_{1}\left(\mathbb{T}^{2}, \psi^{-1}(x)\right)\right)$ est un sous-groupe de rang 2 de $\pi_{1}(V, x)$, alors il existe un plongement $\phi^{\prime}$ isotope à $\phi$ tel que $\phi^{\prime}\left(\mathbb{T}^{2}\right) \cap \psi\left(\mathbb{T}^{2}\right)=\emptyset$.

Démonstration. - On effectue une première isotopie de $\phi$ pour rendre $F$ et $G$ transverses. On raisonne alors par récurrence sur le nombre de composantes de $F \cap G$.

On élimine tout d'abord les composantes contractiles de $F \cap G$ à l'aide de la méthode décrite dans la démonstration du lemme 2.11.

On note ensuite $V^{\prime}$ le revêtement de $V$ de groupe $\phi_{*}\left(\pi_{1}\left(\mathbb{T}^{2}\right)\right) \cap \psi_{*}\left(\pi_{1}\left(\mathbb{T}^{2}\right)\right)$. Les tores $F$ et $G$ se relèvent dans $V^{\prime}$ en des tores $F^{\prime}$ et $G^{\prime}$ pour lesquels $\pi_{1}\left(F^{\prime}\right)=\pi_{1}\left(G^{\prime}\right)=\pi_{1}\left(V^{\prime}\right)$. Comme $V$ est revêtue par $\mathbb{R}^{3}$, la variété $V^{\prime}$ est irréductible et donc $F^{\prime}$ est homotope à $G^{\prime}$. Cette homotopie fournit dans $V$ une application continue $f: \mathbb{T}^{2} \times[0,1] \rightarrow V$ qui vérifie les hypothèses du théorème 2.10 pour $F$ et $G$. Ainsi, il existe $\widetilde{F} \subset F$ et $\widetilde{G} \subset G$ qui sont parallèles dans $V \imath(F \cup G)$ (ce ne sont pas des disques). Une isotopie de $\phi$ qui pousse $\widetilde{F}$ à travers $\widetilde{G}$ permet alors d'éliminer la composante d'intersection de $F$ et $G$ donnée par $\partial \widetilde{F}=\partial \widetilde{G}$ sans en ajouter de nouvelle.

En itérant le procédé, on obtient une isotopie de disjonction de $F$ et $G$. 
Lemme 2.15. - Si $V$ est revêtue par $\mathbb{R}^{3}$ et si $\phi, \psi: \mathbb{T}^{2} \rightarrow V$ sont deux plongements incompressibles du tore qui ont une intersection persistante, on a les propriétés suivantes :

(a) Il existe une courbe fermée simple $\gamma$ dans $\mathbb{T}^{2}$, telle que pour tout plongement $\phi^{\prime}$ isotope à $\phi$, la courbe $\psi(\gamma)$ rencontre $\phi^{\prime}\left(\mathbb{T}^{2}\right)$. Une telle courbe est dite associée à $\phi$ et $\psi$. De plus, si $\alpha$ est une composante non contractile de $\phi\left(\mathbb{T}^{2}\right) \cap \psi\left(\mathbb{T}^{2}\right)$ et si $x \in \psi(\gamma) \cap \alpha$, la classe de $\psi(\gamma)$ dans $\pi_{1}(V, x)$ n'appartient pas à $\phi_{*}\left(\pi_{1}\left(\mathbb{T}^{2}, \phi^{-1}(x)\right)\right)$. Toute courbe fermée simple $\gamma^{\prime} \subset \mathbb{T}^{2}$ qui possède une intersection homologique non nulle avec $\psi^{-1}(\alpha)$ est associée $\grave{a} \phi$ et $\psi$.

(b) Si P désigne un relevé de $\phi\left(\mathbb{T}^{2}\right)$ dans le revêtement universel $\widetilde{V} \simeq \mathbb{R}^{3}$ de $V$, son image $P^{\prime}$ sous l'action de la classe $[\psi(\gamma)] \in \pi_{1}(V, x)$ de $\psi(\gamma)$ est distincte de P. De plus, $P$ et $P^{\prime}$ bordent dans $\mathbb{R}^{3}$ un produit conjugué à $\mathbb{R}^{2} \times[0,1]$ qui les munit d'une orientation, et $[\psi(\gamma)]$ agit en renversant cette orientation. En particulier, la variété obtenue à partir de ce produit en identifiant $P$ et $P^{\prime}$ par le difféomorphisme induit par l'action de $[\psi(\gamma)]$ est conjuguée à $\mathbb{R}^{2} \times \mathbb{S}^{1}$.

\section{Démonstration. -}

Preuve $d u$ (a). - On suppose, dans un premier temps, que les images de $\phi$ et de $\psi$ sont transverses. On remarque dans un deuxième temps que comme $V$ est irréductible, on peut, à l'aide d'une isotopie de $\phi$, éliminer toutes les composantes homotopiquement triviales de $\phi\left(\mathbb{T}^{2}\right) \cap \psi\left(\mathbb{T}^{2}\right)$ (voir la démonstration du lemme 2.11 ).

Ainsi, comme $\phi$ a une intersection persistante avec $\psi$, au moins une composante $\alpha$ de $\phi\left(\mathbb{T}^{2}\right) \cap \psi\left(\mathbb{T}^{2}\right)$ est non homotope à zéro. On note $\gamma$ une courbe fermée simple dans $\mathbb{T}^{2}$ qui possède une intersection homologique non nulle avec $\psi^{-1}(\alpha)$. Si $x$ est un élément de $\psi(\gamma) \cap \alpha$, on sait par application du lemme 2.14 que, pour tout entier relatif $n$ non nul, l'élément $n[\psi(\gamma)] \in \pi_{1}(V, x)$ n'est pas dans l'image de $\phi_{*}$ (car celle-ci contient déjà $[\alpha]$ ), et que $\phi_{*}\left(\pi_{1}\left(\mathbb{T}^{2}\right)\right) \cap \psi_{*}\left(\pi_{1}\left(\mathbb{T}^{2}\right)\right)=\mathbb{Z}[\alpha]$.

On note alors $p: V^{\prime} \rightarrow V$ le revêtement de $V$ de groupe $\psi_{*}\left(\pi_{1}\left(\mathbb{T}^{2}, \psi^{-1}(x)\right)\right)$ et $T$ un relevé compact de $\psi\left(\mathbb{T}^{2}\right)$ dans $V^{\prime}$ qui passe par un point $y$ relevant $x$. On note $\gamma^{\prime}$ le relevé de $\psi(\gamma)$ contenu dans $T$ et $P$ le relevé de $\phi\left(\mathbb{T}^{2}\right)$ qui passe par $y$. Comme $\phi_{*}\left(\pi_{1}\left(\mathbb{T}^{2}\right)\right) \cap \psi_{*}\left(\pi_{1}\left(\mathbb{T}^{2}\right)\right)=$ $\mathbb{Z}[\alpha]$, la sous-variété $P$ est difféormorphe à $\mathbb{R} \times \mathbb{S}^{1}$.

Si $\phi^{\prime}$ est un plongement isotope à $\phi$ et d'image transverse à celle de $\psi$, on considère le relevé $P^{\prime}$ de $\phi^{\prime}\left(\mathbb{T}^{2}\right)$ obtenu en relevant l'isotopie de $\phi$ à partir de $P$. De même que pour $P$, la sousvariété $P^{\prime}$ est difféomorphe à $\mathbb{R} \times \mathbb{S}^{1}$ et le facteur $\mathbb{S}^{1}$ de $P^{\prime}$ est isotope dans $V^{\prime}$ au relevé $\alpha^{\prime}$ de $\alpha$ contenu dans $T$. De plus, toute composante non contractile $\beta$ de $\phi^{\prime}\left(\mathbb{T}^{2}\right) \cap \psi\left(\mathbb{T}^{2}\right)$ se relève en une composante non contractile $\beta^{\prime}$ de $T \cap P^{\prime}$ qui est nécessairement isotope au facteur $\mathbb{S}^{1}$ de $P^{\prime}$. Elle est donc isotope à $\alpha^{\prime}$ dans $V^{\prime}$. Comme $\pi_{1}\left(V^{\prime}\right)=\mathrm{H}_{1}\left(V^{\prime}, \mathbb{Z}\right)=\mathbb{Z}^{2}$, cette courbe $\beta^{\prime}$ est isotope à $\alpha^{\prime}$ dans $T$. On en déduit qu'elle rencontre $\gamma^{\prime}$ et en particulier, $\gamma$ intersecte $\beta$.

Pour conclure, il suffit de remarquer que si $\phi^{\prime}$ est un plongement isotope à $\phi$ dont l'image ne rencontre pas $\psi(\gamma)$, on peut l'isotoper à un plongement $\phi^{\prime \prime}$, dont l'image est transverse à celle de $\psi$ et ne rencontre pas non plus $\psi(\gamma)$, ce qui contredit l'étude précédente.

Preuve $d u(\mathrm{~b})$. - Les plans $P$ et $P^{\prime}$ sont disjoints car $[\psi(\gamma)]$ n'appartient pas à $\phi_{*} \pi_{1}\left(\mathbb{T}^{2}\right)$. Dès lors, $P$ et $P^{\prime}$ bordent dans $\widetilde{V}$ un produit conjugué à $\mathbb{R}^{2} \times[0,1]$ d'après le lemme 2.13. Par une isotopie de $\psi$, on se ramène au cas où le nombre de composantes de $\phi\left(\mathbb{T}^{2}\right) \cap \psi\left(\mathbb{T}^{2}\right)$ est minimal. En particulier, aucune composante de $\phi\left(\mathbb{T}^{2}\right) \cap \psi\left(\mathbb{T}^{2}\right)$ n'est contractile. Pour poursuivre, on précise que $[\psi(\gamma)]$ agit sur $(\widetilde{V}, y)$, où $y$ est un relevé de $x$, et que $P$ passe par $y$. On note $Q$ le relevé de $\psi\left(\mathbb{T}^{2}\right)$ dans $\widetilde{V}$ qui passe par $y$. $[\psi(\gamma)]$ agit par translation $\operatorname{sur} Q$.

AfFirmation. - L'intersection de P et $Q$ est connexe. 
Démonstration. - On sait déjà que les composantes de $P \cap Q$ sont non compactes. Si $P \cap Q$ possède plusieurs composantes, on note $D_{1}$ et $D_{2}$ deux composantes successives dans $Q$. Elles bordent une bande dans $P$ aussi bien que dans $Q$, qui se rencontrent uniquement le long de leur bord. On déduit d'une application combinée classique $\left(\widetilde{V} \simeq \mathbb{R}^{3}\right)$ du lemme de Dehn et du théorème de la sphère ([15]), que la réunion de ces deux bandes borde un tube infini dans $\widetilde{V}$.

Comme dans [18], une application à répétition du théorème des surfaces parallèles ([18], proposition 3.1) à l'ensemble (fini) des relevés de $\phi\left(\mathbb{T}^{2}\right)$ et $\psi\left(\mathbb{T}^{2}\right)$ qui rencontrent ce tube infini fournit l'existence de deux plans $P^{\prime}, Q^{\prime} \subset \widetilde{V}$ relevant respectivement $\phi\left(\mathbb{T}^{2}\right)$ et $\psi\left(\mathbb{T}^{2}\right)$, et de deux bandes $\widehat{P} \subset P^{\prime}$ et $\widehat{Q} \subset Q^{\prime}$, parallèles dans $\widetilde{V}$, qui bordent un tube infini $U$ dont l'intérieur ne rencontre pas les préimages de $\phi\left(\mathbb{T}^{2}\right)$ et de $\psi\left(\mathbb{T}^{2}\right)$.

Soit $m$ un méridien de $U$ qui rencontre $\widehat{P}$ et $\widehat{Q}$ selon un segment. La projection $\pi: \widetilde{V} \rightarrow V$ envoie $\widehat{P}$ sur un anneau $A \subset \phi\left(\mathbb{T}^{2}\right)$ et $\widehat{Q}$ sur un anneau $A^{\prime} \subset \psi\left(\mathbb{T}^{2}\right)$. Ces deux anneaux sont par construction compris entre deux intersections successives (à la fois dans $\phi\left(\mathbb{T}^{2}\right)$ et $\psi\left(\mathbb{T}^{2}\right)$ ) de $\phi\left(\mathbb{T}^{2}\right)$ et $\psi\left(\mathbb{T}^{2}\right)$, et leur réunion forme donc un tore (singulier le long de $A \cap A^{\prime}$ ). La courbe $\pi(m)$ intersecte exactement une fois chaque composante de $A \cap A^{\prime}$ et est donc non contractile dans $A \cup A^{\prime}$. De plus, $\left.\pi\right|_{m}$ est de degré 1 . Comme $m$ est contractile dans $\widetilde{V}$, la courbe $\pi(m)$ est contractile dans $V$ et donc, par application du lemme de Dehn puis du théorème de la sphère, $A \cup A^{\prime}$ borde un tore plein dans $V$ (on rappelle que $V$ est irréductible). Il vient que $A$ et $A^{\prime}$ sont parallèles, ce qui contredit la minimalité du cardinal de $\phi\left(\mathbb{T}^{2}\right) \cap \psi\left(\mathbb{T}^{2}\right)$.

On déduit de cette affirmation que l'intersection de $P^{\prime}$ et $Q$ est connexe. En particulier, l'intersection de $Q$ avec le produit bordé par $P$ et $P^{\prime}$ est une bande dans $Q$. L'action de $[\psi(\gamma)]$ envoie un bord de cette bande sur l'autre, et envoie donc un vecteur pointant à l'intérieur du produit en $P$ sur un vecteur pointant à l'extérieur en $P^{\prime}$.

\section{3. Énoncé et preuve des résultats}

Un des objectifs de ce texte est de démontrer la finitude de la torsion dans toute classe d'isotopie normale ( $c f$. définition 1.3) :

THÉORÈME 3.1. - Si $(V, \xi)$ est une variété de contact universellement tendue irréductible et si $C$ est une classe d'isotopie normale, alors $\operatorname{Tor}(V, \xi, C)<\infty$.

Remarque 3.2. - On présume que pour toute classe d'isotopie $C$ de plongements du produit $\mathbb{T}^{2} \times I$ dans une variété de contact (universellement ?) tendue $(V, \xi)$, on a $\operatorname{Tor}(V, \xi, C)<\infty$.

Remarque 3.3. - D'après un résultat de Haken (voir par exemple [15]), le revêtement universel $\widetilde{V}$ d'une variété fermée, irréductible et qui contient un tore incompressible est difféomorphe à $\mathbb{R}^{3}$. Dans ce cas, d'après Y. Eliashberg [7], si $\xi$ est universellement tendue, son rappel $\tilde{\xi}$ dans $\widetilde{V} \simeq \mathbb{R}^{3}=\{(x, y, z)\}$ est conjugué à la structure d'équation $\mathrm{d} z+x \mathrm{~d} y=0$.

Le théorème 3.1 est une conséquence du lemme fondamental suivant :

Lemme 3.4. - Soient $(V, \xi)$ une variété de contact universellement tendue irréductible, $\phi:\left(\mathbb{T}^{2} \times[0,2 \pi], \xi_{k}\right) \rightarrow(V, \xi)$ un plongement de contact, $\psi: \mathbb{T}^{2} \rightarrow V$ un plongement ayant une intersection persistante avec $\phi$ et $\gamma$ une courbe associée à $\phi$ et $\psi$. Si la courbe $\psi(\gamma)$ est legendrienne, on a $\operatorname{tb}\left(\psi(\gamma), \psi\left(\mathbb{T}^{2}\right)\right) \leqslant-k$.

Voici tout d'abord comment le lemme 3.4 implique le théorème 3.1. 
Démonstration. - On sait que $C$ est une classe d'isotopie normale. Soit donc $\psi: \mathbb{T}^{2} \rightarrow V$ un plongement incompressible du tore tel que tout plongement $\phi$ dans $C$ ait une intersection persistante avec $\psi$, et $\gamma$ une courbe associée à $\phi \in C$ et $\psi$. Quitte à effectuer une isotopie de $\psi$, on suppose que $\psi(\gamma)$ est une courbe legendrienne. Dès lors, d'après le lemme 3.4, si $\phi:\left(\mathbb{T}^{2} \times I, \xi_{n}\right) \rightarrow(V, \xi)$ est un plongement de contact dans $C$, on a :

$$
n \leqslant-\operatorname{tb}\left(\psi(\gamma), \psi\left(\mathbb{T}^{2}\right)\right) .
$$

THÉORÈME 3.5. - Soient $(V, \xi)$ une variété de contact irréductible et universellement tendue et $\phi:\left(\mathbb{T}^{2} \times I, \xi_{1}\right) \rightarrow(V, \xi)$ un plongement de contact. Si $\psi: \mathbb{T}^{2} \rightarrow V$ est un plongement incompressible qui possède une intersection persistante avec $\phi$, alors aucun tore de $V$ isotope à l'image de $\psi$ n'est pré-lagrangien.

Démonstration. - Il est bien connu qu'un tore pré-lagrangien dans $V$ porte un feuilletage caractéristique linéaire et possède donc un voisinage dans $(V, \xi)$ conjugué à $\left(\mathbb{T}^{2} \times\left[\theta_{0}-\varepsilon\right.\right.$, $\left.\left.\theta_{0}+\varepsilon\right], \operatorname{ker}(\cos \theta \mathrm{d} x+\sin \theta \mathrm{d} y)\right)$ par un plongement $F$. En particulier, il existe une valeur de $\theta \in\left[\theta_{0}-\varepsilon, \theta_{0}+\varepsilon\right]$ telle que le feuilletage de $\mathbb{T}^{2} \times\{\theta\}$ soit un feuilletage en cercles et que toute feuille $\gamma$ de ce feuilletage soit associée à $\phi$ et $\psi$ ( $c f$. lemme 2.15). Dès lors, si l'image de $F$ est isotope à celle de $\psi$, on a, par application du lemme 3.4, $\operatorname{tb}\left(F(\gamma), F\left(\mathbb{T}^{2} \times\{\theta\}\right)\right) \leqslant-1$, ce qui contredit le fait que $\gamma$ est une feuille.

On déduit directement de la conjonction des théorèmes 3.1 et 3.5 le résultat de rigidité suivant :

THÉORÈME 3.6. - Si $(V, \xi)$ est une variété de contact fermée, irréductible et universellement tendue, il n'existe qu'un nombre fini de classes d'isotopies de sous-variétés de $V$ difféomorphes à $\mathbb{T}^{2} \times I$ dans lesquelles on puisse trouver une sous-variété de contact conjuguée à $\left(\mathbb{T}^{2} \times[0,2 \pi], \xi_{1}\right)$.

Démonstration. - On commence par appliquer une remarque de E. Giroux : si $\phi$ est un plongement de contact de $\left(\mathbb{T}^{2} \times[0,2 \pi], \xi_{1}\right)$ dans $(V, \xi)$, comme $\xi$ est tendue, nécessairement $\phi\left(\mathbb{T}^{2} \times\{0\}\right)$ est incompressible. On observe ensuite que si $\phi, \psi:\left(\mathbb{T}^{2} \times I, \xi_{1}\right) \rightarrow(V, \xi)$ sont deux plongements de contact, alors $\psi\left(\mathbb{T}^{2} \times\{0\}\right)$ est pré-lagrangien et, par application du théorème 3.5 , les images de $\phi$ et $\psi$ peuvent être disjointes par une isotopie de $\psi$.

Dès lors, si $\left(\phi_{k}\right)_{1 \leqslant k \leqslant n}$ est une suite de plongements de $\left(\mathbb{T}^{2} \times I, \xi_{1}\right)$ dans $(V, \xi)$, on commence par disjoindre, pour $i \geqslant 2$, l'image de $\phi_{i}$ de celle de $\phi_{1}$ (par une suite d'isotopies qui ne préservent pas, bien entendu, la structure $\xi$ ). Par application du lemme 2.11, on disjoint alors, pour $i \geqslant 3$, l'image de $\phi_{i}$ de celle de $\phi_{2}$ dans $V \backslash \phi_{1}\left(\mathbb{T}^{2} \times I\right)$, puis on conclut par récurrence que les images des $\phi_{i}$ peuvent être disjointes dans leur ensemble.

Pour achever la démonstration, il reste à rappeler un résultat de Kneser et Haken (voir par exemple [16]) : pour toute variété fermée et irréductible $V$ de dimension 3 , il existe un entier $n_{V}$, tel que si $p>n_{V}$, toute collection de $p$ surfaces incompressibles disjointes dans $V$ comprend au moins deux surfaces qui bordent un produit. Ici, on obtient directement qu'il existe au plus $n_{V}$ classes d'isotopie de sous-variétés de contact de $(V, \xi)$ conjuguées à $\left(\mathbb{T}^{2} \times I, \xi_{1}\right)$.

COROLlaire 3.7. - Si $(V, \xi)$ est une variété de contact universellement tendue irréductible, pour laquelle il existe un plongement normal $\phi$ de $\mathbb{T}^{2}$ d'image pré-lagrangienne, alors il existe sur $V$ une infinité de structures de contact universellement tendues, deux à deux homotopes comme champs de plans mais deux à deux non isomorphes.

Démonstration. - Comme $\phi$ est d'image pré-lagrangienne, il s'étend en un plongement de contact $\phi^{\prime}:\left(\mathbb{T}^{2} \times\left[-\varepsilon+\theta_{0}, \theta_{0}+\varepsilon\right], \operatorname{ker}(\cos \theta \mathrm{d} x+\sin \theta \mathrm{d} y)\right) \rightarrow(V, \xi), \phi\left(\mathbb{T}^{2}\right)=\phi^{\prime}\left(\mathbb{T}^{2} \times\left\{\theta_{0}\right\}\right)$. Soit $f:\left[-\varepsilon+\theta_{0}, \theta_{0}+\varepsilon\right] \rightarrow \mathbb{R}$ une fonction dont la dérivée est strictement positive et telle 
que $f(t)=t$ près de $\theta_{0}-\varepsilon$ et $f(t)=t+2 n \pi$ près de $\theta_{0}+\varepsilon(n \in \mathbb{N})$. Par application du théorème de recollement 2.8 , la structure $\xi^{\prime}$ obtenue sur $V$ en prolongeant la restriction de $\xi$ au complémentaire de l'image de $\phi^{\prime}$ par $\phi_{*}^{\prime}(\operatorname{ker}(\cos f(\theta) \mathrm{d} x+\sin f(\theta) \mathrm{d} y))$ est universellement tendue (on peut également trouver un difféomorphisme explicite qui conjugue le revêtement universel de $(V, \xi)$ à celui de $\left.\left(V, \xi^{\prime}\right)\right)$. L'homotopie de champs de plans donnée par la famille $(\operatorname{ker}(t(\cos \theta \mathrm{d} x+\sin \theta \mathrm{d} y)+(1-t)(\cos f(\theta) \mathrm{d} x+\sin (\theta) \mathrm{d} y)+t(1-t) \chi \mathrm{d} \theta))_{t \in[0,1]}$, où $\chi: \mathbb{T}^{2} \times\left[-\varepsilon+\theta_{0}, \theta_{0}+\varepsilon\right] \rightarrow[0,1]$ vaut 0 près du bord où les structures coïncident et 1 partout où elles diffèrent, s'étend dans $V$ en une homotopie entre $\xi$ et $\xi^{\prime}$. Par reparamétrisation de $\phi^{\prime}$, on obtient de plus que : $\operatorname{Tor}\left(V, \xi^{\prime}, C\right) \geqslant n$.

D'après le corollaire 3.6, il n'y a qu'un nombre fini de classes d'isotopie de sous-variétés de $V$ qui ont une torsion non nulle et parmi celles-ci, les classes normales ont une torsion finie. Si $n$ est choisi supérieur à $\sup _{C}\{\operatorname{Tor}(V, \xi, C), C$ normale $\}$, on est assuré que $\left(V, \xi^{\prime}\right)$ n'est pas isomorphe à $(V, \xi)$. On peut ainsi construire une famille de structures de contact universellement tendues homotopes $\left(\eta_{k}\right)_{k \in \mathbb{N}}$ sur $V$ pour laquelle la suite $\sup _{C}\left\{\operatorname{Tor}\left(V, \eta_{k}, C\right), C\right.$ normale $\}$ est strictement croissante. Les membres de cette famille sont alors deux à deux non isomorphes et homotopes.

Les théorèmes 1.9 et 1.10 se déduisent immédiatement du théorème d'existence 1.8 et du corollaire 3.7.

On discute à présent le cas des variétés non irréductibles et le comportement de la torsion vis-à-vis des chirurgies d'indice un.

On se base sur le résultat suivant :

THÉORÈME 3.8 ([3]). - Toute variété obtenue par chirurgie d'indice un sur une variété de contact tendue $(V, \xi)$ est naturellement un variété de contact tendue.

Ceci signifie notamment que si $g_{1}, g_{2}: \mathrm{B}^{3} \rightarrow V$ sont deux plongements d'images disjointes tels que $g_{2}^{-1} \circ g_{1}$ renverse l'orientation, alors il existe une structure de contact $\eta$ sur $\mathbb{S}^{2} \times[0,1]$ telle que sur la variété $V^{\prime}$, construite en recollant $\mathbb{S}^{2} \times[0,1]$ à $V \backslash\left(\operatorname{Int}\left(g_{1}\left(\mathrm{~B}^{3}\right)\right) \cup \operatorname{Int}\left(g_{2}\left(\mathrm{~B}^{3}\right)\right)\right)$ par les difféomorphismes $\left.g_{i}\right|_{\mathbb{S}^{2} \times\{i-1\}=\partial \mathrm{B}^{3}}$, la structure $\eta$ se recolle à $\xi$ pour donner une structure tendue $\xi^{\prime}$ qui ne dépend que de la classe d'isotopie de $g_{1}$ et $g_{2}$.

En particulier, si $\phi: \mathbb{T}^{2} \times I \rightarrow V$ est un plongement, dont la classe d'isotopie est $C$ et dont l'image évite $g_{1}\left(\mathrm{~B}^{3}\right) \cup g_{2}\left(\mathrm{~B}^{3}\right)$, il induit un plongement $\phi^{\prime}: \mathbb{T}^{2} \times I \rightarrow V^{\prime}$ de classe d'isotopie $C^{\prime}$ et on a :

$$
\operatorname{Tor}\left(V^{\prime}, \xi^{\prime}, C^{\prime}\right)=\operatorname{Tor}(V, \xi, C) \text {. }
$$

On observe en parallèle que, si $\xi$ et $\zeta$ sont deux structures (universellement) tendues non isotopes sur une même variété $V$ et si $V^{\prime}$ est obtenue par chirurgie d'indice un sur $V$ (par exemple par somme connexe si $V$ n'est pas connexe), alors les structures canoniquement produites sur $V^{\prime}$ à partir de $\xi$ et $\zeta$ sont (universellement) tendues et non isotopes (voir [2], [3]).

Au lieu d'étudier des plongements, pour le plus grand entier $n$ possible, de $\left(\mathbb{T}^{2} \times I, \xi_{n}\right)$ dans une variété de contact $(V, \xi)$, on peut aussi essayer de plonger de manière disjointe, dans une même classe d'isotopie, le plus grand nombre possible de produits $\left(\mathbb{T}^{2} \times I, \xi_{1}\right)$. Les mêmes arguments que ceux développés dans cet article permettent de montrer le résultat suivant :

THÉORÈME 3.9. - Si $(V, \xi)$ est une variété de contact fermée, irréductible et universellement tendue, et $C$ est une classe d'isotopie normale, il existe un entier $n$ tel que toute famille de plongements $\left(\phi_{i}:\left(\mathbb{T}^{2} \times I, \xi_{1}\right) \rightarrow(V, \xi)\right)_{i \in J}$ dont les images sont dans $C$ et deux à deux disjointes soit de cardinal inférieur à $n$. 


\section{Démonstration du lemme 3.4}

\subsection{Schéma de la preuve}

La démonstration du lemme 3.4 comporte deux étapes principales.

On construit d'abord à partir de $(V, \xi)$ et de $\psi$ une variété de contact universellement tendue $(\widehat{W}, \widehat{\xi})$ et un plongement incompressible

$$
\widehat{\psi}: \mathbb{T}^{2} \longrightarrow(\widehat{W}, \widehat{\xi}),
$$

tels que la courbe $\widehat{\psi}\left(\{0\} \times \mathbb{S}^{1}\right)$ soit legendrienne et que :

$$
\operatorname{tb}\left(\psi(\gamma), \psi\left(\mathbb{T}^{2}\right)\right) \leqslant \operatorname{tb}\left(\widehat{\psi}\left(\{0\} \times \mathbb{S}^{1}\right), \widehat{\psi}\left(\mathbb{T}^{2}\right)\right)
$$

(où $\gamma$ est une courbe legendrienne associée à $\phi$ et $\psi$ ). De plus, la construction donne essentiellement l'existence d'un plongement de contact

$$
\left(\mathbb{R} \times \mathbb{S}^{1} \times[0,2 \pi], \operatorname{ker}(\cos k t \mathrm{~d} x+\sin k t \mathrm{~d} y)\right) \longrightarrow(\widehat{W}, \widehat{\xi}),
$$

dont l'image intersecte $\widehat{\psi}\left(\{0\} \times \mathbb{S}^{1}\right)$ selon un segment connexe qui joint un bord de l'image de $\mathbb{R} \times \mathbb{S}^{1} \times[0,2 \pi]$ à l'autre.

On montre ensuite, à l'aide des lemmes 2.1 et 2.2, que, dans cette situation,

$$
\operatorname{tb}\left(\widehat{\psi}\left(\{0\} \times \mathbb{S}^{1}\right), \widehat{\psi}\left(\mathbb{T}^{2}\right)\right) \leqslant-k,
$$

et donc que

$$
\operatorname{tb}\left(\psi(\gamma), \psi\left(\mathbb{T}^{2}\right)\right) \leqslant-k
$$

\subsection{Construction de $(\widehat{W}, \widehat{\xi})$}

Deux structures de contact qui coïncident le long d'une surface fermée $S$ sont isotopes sur un voisinage $U$ de $S$ par une isotopie induisant l'identité sur $S$ (voir [12]). On observe donc tout d'abord qu'il existe $\varepsilon>0$, tel que $\phi$ se prolonge en un plongement de contact (noté à nouveau $\phi$ ) :

$$
\phi:\left(\mathbb{T}^{2} \times[-\varepsilon, 2 \pi+\varepsilon], \operatorname{ker}(\cos k t \mathrm{~d} x+\sin k t \mathrm{~d} y)\right) \longrightarrow(V, \xi) .
$$

Par une isotopie de $\psi$ qui induit une isotopie legendrienne de $\psi(\gamma)$, on se ramène au cas où les images de $\phi$ et $\psi$ sont transverses. On note $\pi:(\tilde{V}, \tilde{\xi}) \rightarrow(V, \xi)$ le revêtement universel de $(V, \xi), Q$ un relevé de $\psi\left(\mathbb{T}^{2}\right)$ dans $\widetilde{V}$ et $\alpha$ un relevé de $\psi(\gamma)$ inclus dans $Q$. Soit de plus un point $x$, contenu dans une composante non homotope à zéro $\eta$ de $\phi\left(\mathbb{T}^{2} \times\{0\}\right) \cap \psi\left(\mathbb{T}^{2}\right)$ ainsi que dans $\psi(\gamma)$. On note alors $x_{1}$ et $x_{2}$ deux relevés successifs de $x$ inclus dans $\alpha$ et $P_{1}$ et $P_{2}$ les deux relevés de $\phi\left(\mathbb{T}^{2} \times\{0\}\right.$ ) qui contiennent respectivement $x_{1}$ et $x_{2}$ (qui sont distincts par application du lemme 2.15). Comme $\phi$ et $\psi$ sont deux plongements incompressibles, $P_{1}, P_{2}$ et $Q$ sont des plans proprement plongés dans $\widetilde{V}$.

On note $W$ la composante de $\widetilde{V} \imath\left(P_{1} \cup P_{2}\right)$ qui contient à la fois $P_{1}$ et $P_{2}$. Comme $\widetilde{V}$ est difféomorphe à $\mathbb{R}^{3}, W$ est conjuguée à $\mathbb{R}^{2} \times[0,1]$ par application du lemme 2.13.

On considère enfin le relevé $A_{1}$ de $\phi\left(\mathbb{T}^{2} \times[-\varepsilon, \varepsilon]\right)$ qui contient $P_{1}$ et $\tau$ le difféomorphisme de $(\widetilde{V}, \tilde{\xi})$ donné par l'action de $[\psi(\gamma)] \in \pi_{1}(V, x)$ sur $\left(\widetilde{V}, x_{1}\right)$ (on oriente $\gamma$ afin que $\tau\left(P_{1}\right)=P_{2}$ ). 


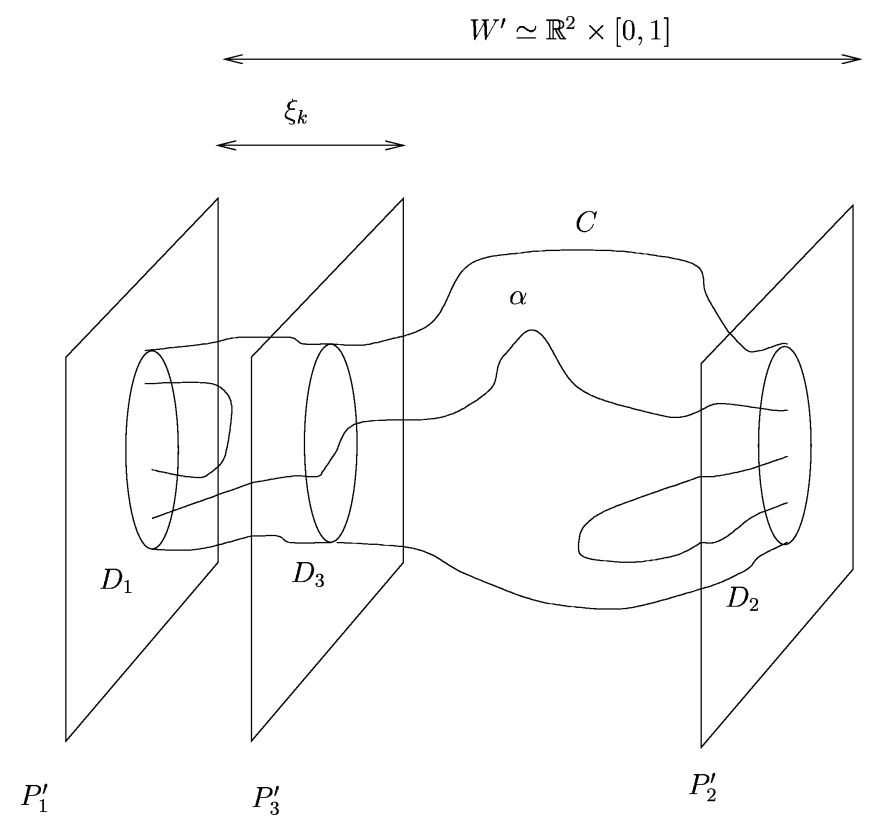

Fig. 2. La variété $W^{\prime}$.

Quitte à échanger le rôle de $P_{1}$ et $P_{2}$, on suppose que la composante de $\pi^{-1}\left(\phi\left(\mathbb{T}^{2} \times[0,2 \pi]\right)\right)$ qui contient $P_{1}$ est incluse dans $W$.

Notation.-Pour la suite, la notation $A+2 \pi$ sous-entend que $A$ est inclus dans $\pi^{-1}\left(\phi\left(\mathbb{T}^{2} \times[-\varepsilon, \varepsilon]\right)\right) \subset \pi^{-1}\left(\phi\left(\mathbb{T}^{2} \times[-\varepsilon, 2 \pi+\varepsilon]\right)\right)$ (ou dans un quotient de cette préimage) et désigne l'image de $A$ par la translation de longueur $2 \pi$ suivant la direction verticale (dirigée $\operatorname{par} \partial / \partial t$ ) dans ces coordonnées. De la même manière, on donne un sens à l'expression $A-2 \pi$.

On applique le lemme 2.6 au compact $\left(\alpha \cap A_{1}\right) \cup\left(\left(\alpha \cap\left(A_{1}+2 \pi\right)\right)-2 \pi\right) \cup\left(\tau^{-1}\left(\alpha \cap \tau\left(A_{1}\right)\right)\right)$ de $A_{1}$ et au plan $P_{1}$ : il existe une isotopie de $\widetilde{V}$ à support compact $K_{1} \subset A_{1}$, qui déforme $P_{1}$ en un plan $P_{1}^{\prime}$, et un disque $D_{1} \subset P_{1}^{\prime}$ qui vérifie :

- $\partial D_{1}$ est transverse à $\tilde{\xi}$ et $\tilde{\xi} D_{1}$ ne contient qu' une singularité elliptique radiale positive ;

- $\alpha \cap P_{1}^{\prime} \subset D_{1}, \alpha \cap\left(P_{1}^{\prime}+2 \pi\right) \subset\left(D_{1}+2 \pi\right)$ et $\alpha \cap \tau\left(P_{1}^{\prime}\right) \subset \tau\left(D_{1}\right)$.

On rappelle de plus que par construction, la structure $\tilde{\xi}$ trace sur $P_{1}^{\prime}$ le feuilletage représenté dans la figure 1 . On note alors :

- $P_{3}^{\prime}=P_{1}^{\prime}+2 \pi \subset W, D_{3}=D_{1}+2 \pi$ et $K_{3}=K_{1}+2 \pi$;

- $P_{2}^{\prime}=\tau\left(P_{1}^{\prime}\right), D_{2}=\tau\left(D_{1}\right)$ et $K_{2}=\tau\left(K_{1}\right)$;

- $W^{\prime} \simeq \mathbb{R}^{2} \times[0,1]$ la variété bordée par $P_{1}^{\prime}$ et $P_{2}^{\prime}$ (figure 2 ).

Soit $C$ un anneau plongé dans $W^{\prime}$ avec les propriétés suivantes :

- pour $i=1,2,3, C \cap P_{i}^{\prime}=\partial D_{i}$;

- la boule $B$ bordée dans $W^{\prime}$ par la sphère $D_{1} \cup C \cup D_{2}$ contient $\alpha \cap W^{\prime}$.

Quitte à déformer $C$ par une isotopie $\mathrm{C}^{0}$-petite fixe près de $\bigcup_{i} \partial D_{i}$, on exige également que :

- le feuilletage caractéristique de $C$ soit non singulier, transverse à $\partial D_{3}$ et que toute feuille de $\tilde{\xi} C$ aille d'un bord de $C$ à l'autre (on applique le lemme 2.4 de E. Giroux pour déformer l'anneau initial entre $\partial D_{1}$ et $\partial D_{3}$ et entre $\partial D_{3}$ et $\left.\partial D_{2}\right)$;

- dans la variété obtenue à partir de $W^{\prime}$ en identifiant $P_{1}^{\prime}$ et $P_{2}^{\prime}$ par $\tau$ (conjuguée à $\mathbb{R}^{2} \times \mathbb{S}^{1}$ d'après le lemme 2.15), le tore donné par l'image de $C$ soit lisse et son feuilletage 
caractéristique contienne une orbite périodique (appliquer le lemme 2.5 pour déformer l'anneau obtenu après la première opération).

On construit à présent la variété $(\widehat{W}, \widehat{\xi})$.

On note $G=\mathbb{Z}[\eta] \subset \pi_{1}(V, x)$ le sous-groupe de $\pi_{1}(V, x)$, isomorphe à $\mathbb{Z}$, engendré par $[\eta]$ (on rappelle que $\eta$ est la composante non homotope à zéro de $\phi\left(\mathbb{T}^{2} \times\{0\}\right) \cap \psi\left(\mathbb{T}^{2}\right)$ qui passe par $x)$. Le groupe $\pi_{1}(V, x)$ agit proprement $\operatorname{sur}\left(\widetilde{V}, x_{1}\right)$, ce qui implique que si $n \in \mathbb{N}$ est choisi suffisamment grand, pour tout $g \in G \backslash\{e\}, n g \cdot B \cap B=\emptyset$ et $n g \cdot K_{1} \cap K_{1}=\emptyset$. On fixe un tel entier $n$.

La variété $(\widehat{W}, \widehat{\xi})$ est obtenue à partir du revêtement $p: \widetilde{V} \rightarrow \widetilde{V} / n G$ de groupe $n G$, et plus précisément à partir de l'image $p(W)$ de $W$. On décrit les images par $p$ des différents objets obtenus précédemment.

Le quotient $p(Q)$ est un revêtement de $\psi\left(\mathbb{T}^{2}\right)$ difféomorphe à $\mathbb{R} \times \mathbb{S}^{1}$ et donc, pour $i=1,2,3$, chaque composante non compacte de $P_{i} \cap Q$ est envoyée par $p$ sur une courbe isotope au facteur $\mathbb{S}^{1}$ donné par l'identification $p(Q) \cong \mathbb{R} \times \mathbb{S}^{1}$. Ainsi, les images des plans $P_{i}$ par $p$ sont des revêtements de $\phi\left(\mathbb{T}^{2} \times\{*\}\right)$ difféomorphes à $\mathbb{R} \times \mathbb{S}^{1}$, où les images par $p$ des relevés de $\eta$ par $\pi$ sont isotopes au facteur $\mathbb{S}^{1}$.

Pour $i=1,2,3$, l'application $p$ restreinte à $K_{i} \cap P_{i}^{\prime}$ est injective car, comme c'est le cas pour $K_{1}$, on remarque que pour tout $g \in G \backslash\{e\}, n g \cdot K_{i} \cap K_{i}=\emptyset$.

On note $P_{i}^{\prime \prime}$ l'anneau infini (conjugué à $\mathbb{S}^{1} \times \mathbb{R}$ ) obtenu en remplaçant dans l'anneau $p\left(P_{i}\right)$ le compact $p\left(K_{i} \cap P_{i}\right)$ par $p\left(K_{i} \cap P_{i}^{\prime}\right)$ et $D_{i}^{\prime \prime} \subset P_{i}^{\prime \prime}$ l'image par $p$ du disque $D_{i}$.

L'application $\tau$ passe au quotient par l'action de $n G$ et fournit un difféomorphisme $\tau^{\prime \prime}$ qui identifie $P_{1}^{\prime \prime}$ et $P_{2}^{\prime \prime}$.

On note à présent $W^{\prime \prime}$ la variété bordée par $P_{1}^{\prime \prime}$ et $P_{2}^{\prime \prime}$ dans $p(\widetilde{V})$ qui contient $P_{3}^{\prime \prime}$ - et qui est une déformation de $p(W)$ dans $p(\widetilde{V})$ - et $(\widehat{W}, \widehat{\xi})$ la variété de contact obtenue à partir de $\left(W^{\prime \prime}, p_{*} \tilde{\xi}\right)$ en identifiant $P_{1}^{\prime \prime}$ et $P_{2}^{\prime \prime} \operatorname{par} \tau^{\prime \prime}$.

On note de plus, pour $i=1,3, \widehat{P}_{i}$ et $\widehat{D_{i}}$ les images respectives de $P_{i}^{\prime \prime}$ et $D_{i}^{\prime \prime}$ dans $\widehat{W}$ $\left(\widehat{P_{1}}=\widehat{P_{2}}\right.$ et $\left.\widehat{D_{1}}=\widehat{D_{2}}\right)$. L'application $\tau^{\prime \prime}$ identifie $D_{1}^{\prime \prime}$ et $D_{2}^{\prime \prime}$, si bien que l'image de $p(B)$ dans $\widehat{W}$ est un tore solide $\widehat{B}$ de bord $\widehat{C}$. De même, dans $\widehat{W}$, l'image de $Q \cap W^{\prime}$ est un tore incompressible $\widehat{T}$ et celle de $\alpha \cap W^{\prime}$ est une courbe fermée simple $\widehat{\alpha} \subset \widehat{T}$. Par construction, $\operatorname{tb}(\widehat{\alpha}, \widehat{T})=\operatorname{tb}\left(\psi(\gamma), \psi\left(\mathbb{T}^{2}\right)\right)$.

\section{LEMME 4.1. - La variété $(\widehat{W}, \widehat{\xi})$ est universellement tendue.}

Démonstration. - Dans $(\widehat{W}, \widehat{\xi})$, la sous-variété $\widehat{P_{1}}$ possède par construction un voisinage tubulaire $U$ conjugué à $\left(\mathbb{S}^{1} \times \mathbb{R} \times[-\varepsilon, \varepsilon], \operatorname{ker}(\cos k t \mathrm{~d} x+\sin k t \mathrm{~d} y)\right)$. De plus, la variété $(\widehat{W}, \widehat{\xi})$ est obtenue par recollement de $\left(\widehat{W} \supsetneq \widehat{P_{1}}, \widehat{\xi}\right) \simeq\left(W^{\prime \prime}, p_{*} \tilde{\xi}\right)$ le long de $\widehat{P_{1}}$. Comme $\left(W^{\prime \prime}, p_{*} \tilde{\xi}\right)$ est universellement tendue (son revêtement universel est une sous-variété de $(\widetilde{V}, \tilde{\xi})$ ), on conclut en appliquant le théorème de recollement 2.8 .

\subsection{Estimation de $\operatorname{tb}\left(\psi(\gamma), \psi\left(\mathbb{T}^{2}\right)\right)=\operatorname{tb}(\widehat{\alpha}, \widehat{T})$}

On admet pour l'instant le lemme suivant :

LEMME 4.2. - Il existe une isotopie $\left(f_{t}\right)_{t \in[0,1]}$ de $\widehat{W}$ telle que :

- $f_{1}(\widehat{\alpha})$ soit legendrienne et rencontre $\widehat{P_{1}}$, resp. $\widehat{P_{3}}$, en un (unique) point $y_{1}$, resp. $y_{3}$, avec $y_{3}=y_{1}+2 \pi$

$-\operatorname{tb}(\widehat{\alpha}, \widehat{T}) \leqslant \operatorname{tb}\left(f_{1}(\widehat{\alpha}), f_{1}(\widehat{T})\right)$. 
LEMME 4.3. - Il existe une isotopie $\left(g_{t}\right)_{t \in[0,1]}$ de $\widehat{W}$ qui fixe $f_{1}(\widehat{\alpha})$ et telle que $g_{1}\left(f_{1}(\widehat{T})\right)$ rencontre $\widehat{P_{1}}$, resp. $\widehat{P_{3}}$, transversalement selon une courbe connexe $\widehat{\eta_{1}}$, resp. $\widehat{\eta_{3}}$, avec $\widehat{\eta_{3}}=$ $\widehat{\eta_{1}}+2 \pi$ (condition de compatibilité). Dans les notations du schéma de la preuve, $g_{1} \circ f_{1}$ est, à paramétrisation près, $\widehat{\psi}$.

Démonstration. - Comme $f_{1}(\widehat{\alpha})$ rencontre $\widehat{P_{1}}$ et $\widehat{P_{3}}$ en un seul point, une seule composante de $f_{1}(\widehat{T}) \cap \widehat{P}_{i}, i=1,3$, est non contractile. Comme de plus $\widehat{W}$ est irréductible, on peut éliminer toutes les autres composantes d'intersection par une isotopie laissant fixe $f_{1}(\widehat{\alpha})$ (voir la démonstration du lemme 2.11). Enfin, par une dernière isotopie, on fait en sorte que la condition de compatiblité soit satisfaite.

La variété $\widehat{W}$ est découpée par $\widehat{P_{1}}$ et $\widehat{P_{3}}$ en deux composantes $W_{1}$ et $W_{2}$ et, compte tenu des propriétés précédentes, le tore $g_{1}\left(f_{1}(\widehat{T})\right)$ rencontre $W_{1}$ et $W_{2}$ selon deux anneaux $T^{1}$ et $T^{2}$. Ici, $W_{1}$ désigne la composante incluse dans la partie produit $\left(\mathbb{S}^{1} \times \mathbb{R} \times[-\varepsilon, 2 \pi+\varepsilon], \xi_{k}\right)$. On a alors :

$$
\operatorname{tb}\left(g_{1}\left(f_{1}(\widehat{\alpha})\right), g_{1}\left(f_{1}(\widehat{T})\right)\right)=\frac{1}{2}\left(\operatorname{rot}\left(g_{1}\left(f_{1}(\widehat{\alpha})\right) \cap T^{1}, T^{1}\right)+\operatorname{rot}\left(g_{1}\left(f_{1}(\widehat{\alpha})\right) \cap T^{2}, T^{2}\right)\right),
$$

où $\operatorname{rot}(a, S)$ désigne le nombre de points, comptés algébriquement le long de l'arc legendrien $a$, où le plan de contact et le plan tangent à la surface $S$ coïncident.

On note $W_{1} / \sim$ et $W_{2} / \sim$ les variétés de contact obtenues respectivement à partir de $W_{1}$ et $W_{2}$, en recollant entre elles les composantes de bord de $W_{1}$, resp. $W_{2}$, par la translation de longueur $2 \pi$. Ces opérations sont compatibles avec la structure de contact et, d'après la condition de compatibilité, transforment $T^{1}$ et $T^{2}$ en deux tores et $g_{1}\left(f_{1}(\widehat{\alpha})\right) \cap W_{1}$ et $g_{1}\left(f_{1}(\widehat{\alpha})\right) \cap W_{2}$ en deux courbes legendriennes fermées simples, incluses dans les deux tores précédents.

Par construction, la variété de contact $W_{1} / \sim$ est conjuguée à $\left(\mathbb{S}^{1} \times \mathbb{R} \times \mathbb{S}^{1}, \xi_{k}\right)$. De plus, la variété de contact $W_{2} / \sim$ est universellement tendue. En effet, par construction, un voisinage de l'image de $\widehat{P_{3}}$ dans $W_{2} / \sim$ est donné par un plongement propre de

$$
U^{\prime}=\left(\mathbb{S}^{1} \times \mathbb{R} \times\left[-\varepsilon^{\prime}, \varepsilon^{\prime}\right], \operatorname{ker}(\cos k t \mathrm{~d} x+\sin k t \mathrm{~d} y)\right)
$$

( $\varepsilon^{\prime}$ est pris très légèrement inférieur à $\varepsilon$, de telle sorte que $\left.P_{1}^{\prime} \subset \pi^{-1}\left(\phi\left(\mathbb{T}^{2} \times\left[-\varepsilon^{\prime}, \varepsilon^{\prime}\right]\right)\right)\right)$. Comme $\left(W_{2}, \xi^{\prime}\right)$ est universellement tendue, on peut appliquer le théorème de recollement 2.8 .

Ainsi, par application du lemme 2.1 à l'image de $\left(g_{1}\left(f_{1}(\widehat{\alpha})\right) \cap T^{1}, T^{1}\right)$ dans $W_{1} / \sim$ et du lemme 2.2 à l'image de $\left(g_{1}\left(f_{1}(\widehat{\alpha})\right) \cap T^{2}, T^{2}\right)$ dans $W_{2} / \sim$, on obtient respectivement :

$$
\frac{1}{2} \operatorname{rot}\left(g_{1}\left(f_{1}(\widehat{\alpha})\right) \cap T^{1}, T^{1}\right) \leqslant-k \quad \text { et } \quad \frac{1}{2} \operatorname{rot}\left(g_{1}\left(f_{1}(\widehat{\alpha})\right) \cap T^{2}, T^{2}\right) \leqslant 0 .
$$

On a donc :

$$
\operatorname{tb}\left(\psi(\gamma), \psi\left(T^{2}\right)\right) \leqslant \operatorname{tb}\left(\widehat{\beta}, f_{1 / 2}(\widehat{T})\right) \leqslant \operatorname{tb}\left(g_{1}\left(f_{1}(\widehat{\alpha})\right), g_{1}\left(f_{1}(\widehat{T})\right)\right) \leqslant-k,
$$

ce qui termine la démonstration du lemme 3.4.

\section{Preuve du lemme 4.2}

Démonstration. - On commence par prouver l'affirmation suivante :

AFFIRMATION 1. - La courbe $\widehat{\alpha}$ est isotope à l'âme du tore solide $\widehat{B}$ via une famille de courbes incluses dans $\widehat{B}$. 
Démonstration. - On applique le lemme 2.12 à $\widehat{\alpha}, \widehat{T}$ et $\widehat{B}$ dans $\widehat{W}$.

On rappelle pour poursuivre que, par construction de $C$, le feuilletage caractéristique de $\widehat{C}$ contient une orbite périodique $\widehat{\beta}$. Celle-ci intersecte $q$ fois le méridien $\partial \widehat{D_{1}}$ de $\widehat{C}$ avec un signe constant.

Cas où $q=1$ : on pose $y_{1}=\widehat{\beta} \cap \widehat{D_{1}}$ et $y_{3}=y_{1}+2 \pi$. Le feuilletage de $\widehat{P}_{3}$ comprend une singularité elliptique radiale positive $e_{+}$et toutes les feuilles issues de $e_{+}$ont pour ensemble limite une même singularité elliptique négative $e_{-}$, à l'exception de l'une d'entre elles dont l'ensemble limite est composé d'une singularité hyperbolique positive $h_{+}$(voir figure 1). Quitte à effectuer une petite isotopie legendrienne de $\widehat{\beta}$, on suppose que les points $\widehat{\beta} \cap \widehat{D_{3}}$ et $y_{3}$ ne sont pas situés sur la liaison entre $e_{+}$et $h_{+}$. On se donne alors un plongement $F: \mathbb{D}^{2} \times[-1,1] \rightarrow \widehat{W}$ avec les propriétés suivantes :

- $F\left(\mathbb{D}^{2} \times[-1,1]\right) \cap \widehat{P_{3}}=F\left(\mathbb{D}^{2} \times\{0\}\right)$ et $F\left(\mathbb{D}^{2} \times\{0\}\right)$ contient l'ensemble limite de $e_{+}$ dans son intérieur,

- l'image de $F$ ne rencontre pas $\widehat{P_{1}}$,

- la courbe $\widehat{\beta}$ rencontre transversalement chaque disque $F\left(\mathbb{D}^{2} \times\{t\}\right), t \in[0,1]$, en un point.

Dès lors, d'après le lemme 2.7 , il existe une courbe $\widehat{\beta^{\prime}}$ isotope à $\widehat{\beta}$ par une isotopie legendrienne (fixe en dehors de l'image de $F$ ) qui rencontre $\widehat{P_{3}}$ transversalement selon $y_{3}$ (et, bien sûr, $\widehat{P_{1}}$ en $\left.y_{1}\right)$.

D'après l'affirmation 1 , la courbe $\widehat{\alpha}$ est isotope à $\widehat{\beta}$ par la restriction d'une isotopie de $\widehat{W}$ via une famille de courbes qui restent dans le tore solide $\widehat{B}$. On note alors $\left(f_{t}\right)_{t \in[0,1]}$ une isotopie de $X$ qui envoie $\widehat{\alpha}$ sur $\widehat{\beta}^{\prime}$, et telle que $f_{1 / 2}(\widehat{\alpha})=\widehat{\beta}$ (on impose que pour tout $t \in[0,1 / 2], f_{t}(\widehat{\alpha}) \subset \widehat{B}$ ).

D'après le lemme 2.3 , on $\operatorname{tb}(\widehat{\alpha}, \widehat{T}) \leqslant \operatorname{tb}\left(\widehat{\beta}, f_{1 / 2}(\widehat{T})\right)$.

De plus, par construction : $\operatorname{tb}\left(\widehat{\beta}, f_{1 / 2}(\widehat{T})\right)=\operatorname{tb}\left(\widehat{\beta}^{\prime}, f_{1}(\widehat{T})\right)$, et donc

$$
\operatorname{tb}(\widehat{\alpha}, \widehat{T}) \leqslant \operatorname{tb}\left(f_{1}(\widehat{\alpha}), f_{1}(\widehat{T})\right) .
$$

Cas où $q \neq 1$ : on va se ramener au cas où $q=1$.

Soit $y_{1} \in \widehat{D_{1}} \cap \widehat{\beta}$. On note $y_{1}^{\prime}$ le point de premier retour de $\widehat{\beta}$ sur $\widehat{D_{1}}$, obtenu en partant de $y_{1}$, et $f$ la portion de $\widehat{\beta}$ comprise entre $y_{1}$ et $y_{1}^{\prime}$. Par hypothèse, $y_{1} \neq y_{1}^{\prime}$.

Comme le germe d'une structure de contact au voisinage d'une surface est uniquement déterminé par son feuilletage caractéristique, il existe un plongement de contact

$$
g:\left(\{(r \leqslant 1, \theta,-\ell \leqslant z \leqslant \ell)\} \subset \mathbb{R}^{3}=\{(r, \theta, z)\}, \zeta=\operatorname{ker}\left(\mathrm{d} z+r^{2} \mathrm{~d} \theta\right)\right) \longrightarrow(\widehat{W}, \widehat{\xi}),
$$

tel que :

$-g(\{z=0\})=\widehat{D_{1}}$,

$-g(\{r=1\}) \subset \widehat{C}$,

- l'image de $g$ soit incluse dans le tore solide bordé par $\widehat{C}$ dans $\widehat{W}$ et ne rencontre pas $\widehat{P_{3}}$.

On trace alors sur l'anneau $\{r=1\}$ un feuilletage $F$ qui possède les propriétés suivantes :

- les feuilles de $F$ sont transverses à chaque cercle $\{r=1, z=c\}$,

- $F=\zeta\{r=1\}$ sur la réunion d'anneaux $\{r=1,-\ell \leqslant z \leqslant-\ell / 2\} \cup\{r=1, \ell / 2 \leqslant z \leqslant \ell\}$, et les feuilles de $F$ sont transverses au feuilletage $\zeta\{r=1\}$ en dehors de cette réunion, de telle sorte qu'en tout point, le couple formé des vecteurs engendrant respectivement les feuilletages orientés $\zeta\{r=1\}$ et $F$ oriente $\{r=1\}$ comme $(\partial / \partial z, \partial / \partial \theta)$,

- la feuille de $F$ qui part de $g^{-1}(f) \cap\{r=1, z=-\ell\}$ aboutit à $g^{-1}(f) \cap\{r=1, z=\ell\}$ en ayant fait strictement moins d'un tour de méridien de plus que le feuilletage $\zeta\{r=1\}$. 
Le relevé legendrien du feuilletage $F$ dans $\{r \leqslant 1\} \backslash\{r=0\}-$ dont les feuilles sont legendriennes et qui se projette sur $F$ par la projection parallèle à $\partial / \partial r$ - détermine un nouvel anneau dont l'image par $g$ détermine un nouveau tore $\widehat{C}^{\prime} \subset \widehat{B}$ isotope à $\widehat{C}$.

Par construction, la courbe du feuilletage caractéristique de $\widehat{C}^{\prime}$, égale à $f$ en dehors de l'image de $g$, est fermée et intersecte une fois un méridien de $\widehat{C}^{\prime}$. Elle est de plus isotope à $\widehat{\alpha}$ via une famille de courbes incluses dans le tore solide $\widehat{B}$ bordé par $\widehat{C}$. On note $\left(\phi_{t}\right)_{t \in[0,1]}$ une telle isotopie.

AFFIRMATION 2. - On $a \operatorname{tb}(\widehat{\alpha}, \widehat{T}) \leqslant \operatorname{tb}\left(\phi_{1}(\widehat{\alpha}), \phi_{1}(\widehat{T})\right)$.

Démonstration. - On considère le revêtement cyclique d'ordre $q$ de $\widehat{W}$ déterminé par $\widehat{\alpha}$ :

$$
p:(\dot{W}, \dot{\xi}) \longrightarrow(\widehat{W}, \widehat{\xi})
$$

Comme $(\widehat{W}, \widehat{\xi})$ est universellement tendue, $(\dot{W}, \dot{\xi})$ est universellement tendue. La courbe $\widehat{\beta}$ se relève en une courbe $\dot{\beta}$ qui intersecte une fois le méridien du tore $\dot{C}$ relevant $\widehat{C}$ et contenant $\dot{\beta}$. On note $\dot{B}$ le tore plein bordé par $\dot{C}$ dans $\dot{W}$. En appliquant le lemme 2.3 dans ce revêtement, il vient : $\operatorname{tb}(\dot{\alpha}, \dot{T})=q \operatorname{tb}(\widehat{\alpha}, \widehat{T}) \leqslant \operatorname{tb}\left(\dot{\beta}, \dot{T}^{\prime}\right)$, où :

- $\dot{\alpha}$ est le relèvement de $\widehat{\alpha}$ inclus dans le tore plein $\dot{B}$;

- $\dot{T}$ est le relèvement de $\widehat{T}$ qui contient $\dot{\alpha}$;

- $\dot{T}^{\prime}$ est un tore contenant $\dot{\beta}$ et isotope au tore $\dot{T}$, via une isotopie à support dans $\dot{B}$.

Or, par construction, le relèvement de $\phi_{1}(\widehat{\alpha})$ contenu dans $\dot{B}$ est obtenu à partir de $\dot{\beta}$ en lui ajoutant un nombre de spirales autour du méridien de $\dot{C}$ strictement inférieur à $q$. On a donc :

$$
q \operatorname{tb}\left(\phi_{1}(\widehat{\alpha}), \phi_{1}(\widehat{T})\right)+q>\operatorname{tb}\left(\dot{\beta}, \dot{T}^{\prime}\right),
$$

ce qui permet d'obtenir que

$$
\operatorname{tb}(\widehat{\alpha}, \widehat{T})<\operatorname{tb}\left(\phi_{1}(\widehat{\alpha}), \phi_{1}(\widehat{T})\right)+1
$$

On conclut en remarquant qu'il s'agit d'une inégalité entre entiers.

Pour achever la démonstration du lemme 4.2 dans le cas $q \neq 1$, on applique la conclusion du cas $q=1$ au couple $\left(\phi_{1}(\widehat{\alpha}), \phi_{1}(\widehat{T})\right)$ (pour obtenir la propriété $y_{3}=y_{1}+2 \pi$ ).

\section{Remerciements}

Ce travail a été réalisé en grande partie à l'École normale supérieure de Lyon. Il a bénéficié des suggestions d'Emmanuel Giroux et de Jean-Pierre Otal. Je les remercie chaleureusement de leur disponibilité et de leur aide précieuse. Je remercie également le rapporteur de ce texte pour la pertinence de ses remarques.

\section{RÉFÉRENCES}

[1] Bennequin D., Entrelacements et équations de Pfaff, Astérisque 107-108 (1983) 83-161.

[2] Chekanov Y., Contact disjunction in tight three-manifolds, Preprint, 1999.

[3] Colin V., Chirurgies d'indice un et isotopies de sphères dans les variétés de contact tendues, $C$. $R$. Acad. Sci. Paris, Série I 324 (1997) 659-663.

[4] Colin V., Recollement de variétés de contact tendues, Bull. Soc. Math. France 127 (1999) 101-127.

[5] Colin V., Structures de contact tendues sur les variétés toroïdales et approximation de feuilletages sans composante de Reeb, Prépublication 99/11-2 de l'université de Nantes. 
[6] Eliashberg Y., Classification of over-twisted contact structures on 3-manifolds, Invent. Math. 98 (1989) 623-637.

[7] Eliashberg Y., Contact 3-manifolds, twenty years since J. Martinet's work, Ann. Inst. Fourier 42 (1992) 165-192.

[8] Eliashberg Y., Filling by holomorphic discs and its applications, London Math. Soc. Lect. Notes Ser. 151 (1991) 45-67.

[9] Eliashberg Y., Thurston W., Confoliations, Univ. Lect. Ser., Vol. 13, 1998.

[10] Gabai D., Foliations and the topology of 3-manifolds, J. Differ. Geom. 18 (1983) 445-503.

[11] Giroux E., Convexité en topologie de contact, Comment. Math. Helvetici 66 (1991) 18-33.

[12] Giroux E., Topologie de contact en dimension 3, Séminaire Bourbaki 760 (1992-1993) 1-27.

[13] Giroux E., Une structure de contact, même tendue est plus ou moins tordue, Ann. Scient. Éc. Norm. Sup. 27 (1994) 697-705.

[14] Giroux E., Une infinité de structures de contact tendues sur une infinité de variétés, Invent. Math. 135 (1999) 789-802.

[15] Hempel J., Three Manifolds, Ann. Math. Studies, Princeton Univ. Press.

[16] JACO W., Lectures on Three Manifolds Topology, Amer. Math. Soc.

[17] KandA Y., The classification of tight contact structures on the 3-torus, Commun. Anal. Geom. 5 (1997) 413-438.

[18] Waldhausen F., On irreducible 3-manifolds which are sufficiently large, Ann. of Math. 87 (1968) 56-88.

(Manuscrit reçu le 15 décembre 1999; accepté, après révision, le 11 septembre 2000.)

\footnotetext{
Vincent COLIN

Département de mathématiques,

UMR 6629 du CNRS,

Université de Nantes,

2, rue de la Houssinière,

B.P. 92208 ,

44322 Nantes cedex 3, France

E-mail : Vincent.Colin@math.univ-nantes.fr
} 